\title{
Foliar application of micronutrients on essential oils of borago, thyme and marigold
}

\author{
M. Yadegari* \\ Department of Agronomy and Medicinal Plants, Faculty of Agriculture, Shahrekord Branch, Islamic Azad \\ University, Po.Box:166. Shahrekord, Iran. *Corresponding author: mehrabyadegari@gmail.com
}

\begin{abstract}
To study the effect of micronutrients foliar applications in medicinal plants contain of Borago, Calendula and Thyme on yield and essence production, the investigations were performed in complete randomized block design with 3 replications at 2011 and 2012 in Shahrekord, Iran. Results showed that $\mathrm{Fe}^{2+}, \mathrm{Cu}^{2+}, \mathrm{Zn}^{2+}$ and $\mathrm{Mn}^{2+}$ applications on flavonoids, phenols, carotenoids, essence percentage, fresh/dry flower matter, number of flowers, and root/shoot dry/fresh matter in Borago officinalis and Calendula officinalis L.; carvacrol and thymol percentage, essence percentage, number of stem, height, and root/shoot dry/fresh matter in Thymus vulgaris L., the combinations of $400 \mathrm{ppm}$ of $\mathrm{Fe}, \mathrm{Cu}, \mathrm{Zn}$ and $\mathrm{Mn}\left(\mathrm{Fe}_{3} \mathrm{Cu}_{3} \mathrm{Zn}_{3} \mathrm{Mn}_{3}\right)$ produced the greatest amounts in most of measured characters. The most of phenols, carotenoids and flavonoids in Calendula and Borago; thymol and carvacrol in Thyme; weight of dry flower and number of flower per plant in two seasons were produced by $\mathrm{Fe}_{3} \mathrm{Cu}_{3} \mathrm{Zn}_{3} \mathrm{Mn}_{3}$ treatment.
\end{abstract}

Keywords: Essence, medicinal plants, nutrition, phytochemical

\section{Introduction}

Borago officinalis Linn. (Family; Boraginaceae) is a hairyannual herb has been known forits mood elevating properties as early as the first century A.D. (Tyler, 1993). The plant is reputed as antispasmodic, antihypertensive, antipyretic, aphrodisiac, demulcent and is also considered useful to treat asthma, bronchitis, cramps, palpitations and kidney ailments, Optimum, minimum, and maximum temperatures for borage germination are 23,9 , and $37^{\circ} \mathrm{C}$, respectively (Gilbertson et al. 2014). Borage oil has been reported to lower serum cholesterol, phospholipids and triglyceride levels and increases the levels of 6 polyunsaturated fatty acids in the plasma, liver, aorta and renal artery tissues. Dietary use of borage oil exhibited immuno-modulatory and cytotoxic and free radical scavenging activities. Flavonoids and phenolic compounds were the important essential oils in this plant and no have tannin (Lin et al. 2002; Wafa et al. 2011; RatzLyko et al. 2014). Calendula also known marigold is annual herb (Danielski et al. 2007). Calendula seeds contain high concentrations of calendic acid (C18:3) which can be used as tung and linseed oil substitutes. Optimum germination temperature is at 
16-17 ${ }^{\circ} \mathrm{C}$ (Eberle et al. 2014). The vegetative parts of the plant are mid green while the stems are angular and covered in fine hairs. Marigold is cultivated for its flowers with receptacle or flowers without receptacle (Borghei et al.2011) which are used as the medical raw material. Flavonoids, carotenoids and polyphenols are the active ingredient contents reported previously for C.officinalis flowers (Bunghez and Ion, 2011; Lim, 2014). The most essence in this plant is formed at full blooming $(0.97 \%)$ and the least of essence $(0.13 \%)$ before flowering (Sedghi et al. 2011). Thymus vulgaris L. (thyme), a member of the family Lamiaceae, is widely used in Iran folk medicine. Thymus species are commonly used as herbal tea, flavoring agents (condiment and spice) and medicinal plants. The best time for harvesting of this plant is the beginning of flowering period (FriekeKuper et al. 2014). The major components are phenols (mainly thymol and carvacrol), monoterpen hydrocarbon and alcohol that have insecticide activity (Mendes et al. 2014; Deletre et al. 2015). Among these, thymol and carvacrol are the main compounds (Yadegari, 2012; Jamali et al. 2014). Although many studies performed about of macronutrients effects on plants (Quezada et al. 2007; Wyllyam Do Vale et al. 2008; Khan et al. 2014; Raza et al. 2014; Sandana and Pinochet, 2014; Yañez-Mansilla et al. 2015), few studies examining Iron, Copper, Zinc and Manganese fertility in Borago, Calendula and Thyme have been conducted, but they are not specific to the medicinal use of this plant. Iron is one of the three micro essential nutrient elements required by plants. Fe is important in cytochrome structure. The Fe (II) form is normally below the detection level in plants (Schönherr et al. 2005). Copper is an essential microelement in higher plants as it occurs as part of the prosthetic groups of several enzymes (Datta et al. 2011). Zinc is an important micronutrient associated with several enzymatic activities in all photosynthetic plants. $\mathrm{Zn}$ is necessary in vital enzymes and growth regulators (Babaeian et al. 2012; Samia and Mohmoud, 2009).

Orioli Junior et al. (2008) showed that zinc application methods did not influence the initial growth of the wheat plants. The zinc concentration in leaves was influenced by the different application methods, being the leaf pulverization the one that was caused the largest zinc accumulation on aerial part dry matter but Mello Prado et al. (2007) reported that zinc application in the wheat seeds influenced the nutritional stage and plant dry matter production.

Manganese is involved in many biochemical functions, primarily acting as an activator of enzymes such as dehydrogenases and decarboxylases involved in respiration, amino acid and lignin synthesis, and hormone concentrations (Younis et al. 2013). In alkaline soils nutrient concentration may be not enough and therefore micro nutrient in this soil immobilized quickly and roots of plants can't absorb from soil and some of nutrient no transition to leaves, in this places spry application of micro nutrient solve this problem and reduce soil fertilizer loss. Foliar fertilizer is particularly useful technique which can be designed to meet plants specific needs for one or more micro or macro nutrients especially trace minerals and enable to correct deficiencies, strengthen weak or damaged crops, speed growth and grow better (Said-Al Ahl and Mahmoud, 2010). Objectives of this study were to determine the effects of Iron, Zinc, Copper and Manganese foliar applications on vegetative, reproductive and phytochemical characters of three medicinal plants.

\section{Materials and Methods}

\subsection{Plant material and fertilizers}

Seeds of Borago officinalis Linn, C.officinalis var Qazvin and T.vulgaris L. obtained from the PakanBazr 
Company, Isfahan, Iran. Sowing was conducted manual, plant spacing in the each row was $5 \mathrm{~cm}$, and two seeds were put in the soil. In the 3-5 leaves phase plants were thin out to final row distance. During the vegetation, used practical measures were standard (3 earth up and pest protection). Four foliar fertilizers Librel Fe-Lo, Librel $\mathrm{Cu}$, Librel $\mathrm{Zn}$ and Librel $\mathrm{Mn}$ were applied and all of them are mineral fertilizers. Librel Fe-Lo contains $13.2 \%$ chelated iron, Librel $\mathrm{Zn}$ is a foliar fertilizer which contains 14\% Zinc in chelated form, Librel Cu has 14\% copper in chelated form and Librel Mn inclusive of $13 \%$ Mn chelated with EDTA (obtained from The Chemical Company of England and Germany). Theses fertilizers were sprayed at four concentrations $\left(\mathrm{Fe}_{1}, \mathrm{Fe}_{2}, \mathrm{Fe}_{3}, \mathrm{Fe}_{4}\right.$ were $0,200,400$ and 600 ppm of Fe, respectively and similar in other micronutrients).

\subsection{Experimental conditions}

Field trials were established in 2012 and 2013 at Shahrekord $\left(50^{\circ} 56^{\prime}\right.$ E, $32^{\circ} 18^{\prime}$ N) South-Western Iran. The soil (Typiccalcixerocrepts) physical and chemical properties are shown in Table 1. Experiments were arranged in a randomized complete block design with a factorial layout and three replications. All experiments were carried out in triplicate. Top soil of the experimental plot area was kept moist throughout the growing season when necessary. After soil test, the required nutrients were added to soil. At the beginning of the blooming stage, shoots of thyme plants and in the end of the blooming stage, shoots of calendula and borago plants were harvested.

Table 1. Physical and chemical properties of soil for experiment

\begin{tabular}{lcccccccccc}
\hline Year & Clay & Silt & Sand & $\mathrm{N}_{\text {total }}$ & $\mathrm{K}_{\text {available }}$ & $\mathrm{P}_{\text {available }}$ & O.C & $\mathrm{pH}$ & E.C & Depth \\
& $(\%)$ & $(\%)$ & $(\%)$ & $(\%)$ & $(\mathrm{mg} / \mathrm{kg})$ & $(\mathrm{mg} / \mathrm{kg})$ & $(\%)$ & & $\left(\mathrm{ds} . \mathrm{m}^{-1}\right)$ & $(\mathrm{Cm})$ \\
& & & & & & & & & & \\
& & & & & & & & & & \\
\hline 2012 & 39 & 33 & 28 & 0.158 & 741 & 15.9 & 1.13 & 8.05 & 0.76 & $0-30$ \\
\hline 2013 & 39 & 32 & 29 & 0.17 & 720 & 16.2 & 1.2 & 7.89 & 0.73 & $0-30$
\end{tabular}

\subsection{Preparation of extracts from thyme, calendula and borago samples}

Several parameters including dry and fresh weight of shoot and root, number of flower, dry and fresh flower matter and the amount of essence percentage as well as the chemical component of the essence (carotenoids, flavonoids, phenols, thymol and carvacrol) were determined. Thermo Finnegan Trace
$2000 \mathrm{GC} / \mathrm{MS}$, made in the USA, was employed with a HP-5MS capillary column (30 m long and $0.25 \mathrm{~mm}$ wide, and a $0.25 \mu \mathrm{m}$ of film thickness) at a $250^{\circ} \mathrm{C}$ of injector chamber. The initial column temperature was at $120^{\circ} \mathrm{C}$ for $5 \mathrm{~min}$ then raised to $280^{\circ} \mathrm{C}$ at the rate of $10^{\circ} \mathrm{C} / \mathrm{min}$. Helium was used as a carrier gas at a rate of $35 \mathrm{ml} / \mathrm{min}$. MS parameters were as follows: ionization energy, $70 \mathrm{eV}$; ion source temperature, $200{ }^{\circ} \mathrm{C}$, voltage, $3000 \mathrm{v}$; and mass 
range, 30 to 600 . The compositions of the essential oil were identified by comparison of their retention indexes, retention times and mass spectra with those of authentic samples in Wiley library. Harvested flowers were dried at room temperature for 1 week. Flower extracts were obtained by stirring $1 \mathrm{~g}$ of dry flower powder with $10 \mathrm{ml}$ of pure methanol for 30 min. The extracts were then kept for $24 \mathrm{~h}$ at $4{ }^{\circ} \mathrm{C}$, filtered through a Whatman no. 4 filter paper, and evaporated under vacuum to dryness and stored at $4{ }^{\circ} \mathrm{C}$ until analysed. The content of total phenolic of B.officinalis and C.officinalis methanolic extract was determined using the Folin-Ciocalteu $(\mathrm{F}-\mathrm{C})$ reagent. Dried samples were hydrolyzed, slightly modified. $20 \mathrm{ml}$ of methanol containing BHT $\left(1 \mathrm{~g} \mathrm{l}^{-1}\right)$ were added to $0.5 \mathrm{~g}$ of a dried sample. Then $10 \mathrm{ml}$ of $1 \mathrm{M}$ $\mathrm{HCl}$ were added. The mixture was stirred carefully and sonicated for $15 \mathrm{~min}$ and refluxed in a water bath at $90{ }^{\circ} \mathrm{C}$ for $2 \mathrm{~h}$. The obtained mixture was injected to HPLC. The phenolic compounds' analysis was carried out using an Agilent Technologies 1100 series liquid chromatograph (RP-HPLC, Palo Alto, CA) coupled with an UV-vis multiwave length detector. The separation was carried out on a $250 \times 4.6-\mathrm{mm}$, $4 \mu \mathrm{m}$ Hypersil ODS C18 reversed phase column at ambient temperature. The mobile phase consisted of acetonitrile (solvent A) and water with $0.2 \%$ sulphuric acid (solvent B). The flow rate was kept at $0.5 \mathrm{ml} \mathrm{min}{ }^{-1}$. The gradient programme was as follows: $15 \% \mathrm{~A} / 85 \% \mathrm{~B}, 0-12 \mathrm{~min} ; 40 \% \mathrm{~A} / 60 \% \mathrm{~B}$, 12-14min; 60\% A/40\% B, 14-18min; 80\% A/20\% B, 18-20 min; $90 \% \mathrm{~A} / 10 \% \mathrm{~B}, 20-24 \mathrm{~min} ; 100 \% \mathrm{~A}$, 24-28 min. The injection volume was $20 \mu \mathrm{l}$, and peaks were monitored at $280 \mathrm{~nm}$. Samples were filtered through a $0.45 \mu \mathrm{m}$ membrane filter before injection. Peaks were identified by congruent retention times compared with standards. Analyses were performed by triplicate (Adams, 2001).

\subsection{Data analysis}

All data were subjected to ANOVA using the statistical computer package SAS and treatment means separated using Duncan's multiple range test at $P<0.05$ level.

\section{Results}

Results showed the significant differences between treatments. Although in some of single treatments no significant differences, but in combinations and by Duncan's comparison, there were differences in most characters that result the $\mathrm{Fe}_{3} \mathrm{Cu}_{3} \mathrm{Mn}_{3} \mathrm{Zn}_{3}$ treatment was the best (Tables 2,4 ). It seem that by synergist application of micro nutrient results to additive of effectiveness of measured characters but upper than $400 \mathrm{ppm}$, the action of fertilizer was toxicity. The components of borago, calendula and thyme oil were influenced by different treatments. The application treatment of $\mathrm{Fe}, \mathrm{Cu}, \mathrm{Mn}$ and $\mathrm{Zn}$ was significantly improved the growth characters including dry and fresh weight of shoot and root, number of flower, dry and fresh flower matter and the amount of essence percentage as well as the chemical component of the essence (carotenoids, flavonoids, phenols, thymol and carvacrol) were determined showed significant response with increasing rates of foliar fertilizers (Table 2). Meanwhile, the effect of combination of four micronutrients was surpassed that of single of them. Thus, the foliar application of $400 \mathrm{ppm} \mathrm{Fe}, \mathrm{Cu}$, $\mathrm{Mn}$ and $\mathrm{Zn}$ caused the highest increments compared with other treatments. Length of stem in control plants was the least also the number of stem per plant in $\mathrm{Fe}_{4} \mathrm{Cu}_{4} \mathrm{Mn}_{4} \mathrm{Zn}_{4}$ was the least (data no published). In character of number of flowers per plant, control plants and plants treated by treatments of $600 \mathrm{ppm}$ of micronutrients were similar. The application of 400 ppm of $\mathrm{Fe}, \mathrm{Cu}, \mathrm{Mn}$ and $\mathrm{Zn}$ has increased flavonoids, 
phenols and carotenoids in the oil of borago and calendula; thymol and carvacrol in thyme too. However, combination of $\mathrm{Fe}_{3} \mathrm{Cu}_{3} \mathrm{Mn}_{3} \mathrm{Zn}_{3}$ treatment was more effective than other treatments. Most of amount of measured characters (for example number and weight of flower) decreased by application upper than $400 \mathrm{ppm}$ of $\mathrm{Fe}, \mathrm{Cu}, \mathrm{Mn}$ and $\mathrm{Zn}$. Use of the upper range of optimum micro nutrient decreased the yield and essence production. Control treatment in many places made better yield components than $600 \mathrm{ppm}$ concentration of $\mathrm{Fe}, \mathrm{Cu}, \mathrm{Mn}$ and $\mathrm{Zn}$. In most treatments, combination of $\mathrm{Fe}_{3} \mathrm{Cu}_{3} \mathrm{Mn}_{3} \mathrm{~B}_{3}, \mathrm{Fe}_{3} \mathrm{Cu}_{3} \mathrm{Mn}_{2} \mathrm{Zn}_{2}$ and $\mathrm{Fe}_{3} \mathrm{Cu}_{3} \mathrm{Mn}_{1} \mathrm{Zn}$, made the maximum amount of characters, but $\mathrm{Fe}_{3} \mathrm{Cu}_{3} \mathrm{Mn}_{3} \mathrm{~B}_{3}$ was the best combination (Table 4). In most times, control plants made alike of $\mathrm{Fe}_{4} \mathrm{Cu}_{4} \mathrm{Mn}_{2} \mathrm{Zn}_{2}, \mathrm{Fe}_{4} \mathrm{Cu}_{4} \mathrm{Mn}_{3} \mathrm{Zn}_{3}$ and $\mathrm{Fe}_{4} \mathrm{Cu}_{4} \mathrm{Mn}_{4} \mathrm{Zn}_{4}$. For toxicities of upper concentrations of $\mathrm{Fe}, \mathrm{Cu}, \mathrm{Mn}$ and $\mathrm{Zn}$, the results obtained similar by control plants.
It was clear from the presented data that the highest levels of the four foliar fertilizers were more effective than lower levels, and Librel Fe-Lo fertilizer was superior to other micronutrients. However, the highest essence percentage was found with $\mathrm{Fe}_{3} \mathrm{Cu}_{3} \mathrm{Mn}_{3} \mathrm{Zn}_{3}$. Concentration of micronutrients positively affected on characters measured and between the dry and fresh weight of shoot and root, number of flower, dry and fresh flower matter and the amount of essence percentage as well as the chemical component of the essence were determined of medicinal plants were positive correlated and in best combinations of treatments (Table 3) the $\mathrm{Fe}_{3} \mathrm{Cu}_{3} \mathrm{Mn}_{3} \mathrm{Zn}_{3}$ was the best. Essence percentage positively correlated with the weight of shoot dry and fresh matter, number and weight of flowers. Also weight of shoot dry and fresh matter positively correlated with number and weight of flowers and essence percentage of medicinal plants (Table 3).

Table 2. Complex analysis of variance of variation of, flavonoids, phenols, carotenoids, essence percentage, fresh flower matter, dry flower matter, number of flowers, and root/shoot dry/fresh matter in plants of Borago officinalis L.

\begin{tabular}{|c|c|c|c|c|c|c|c|c|c|c|c|c|}
\hline \multirow[t]{2}{*}{ Source of Variation } & \multirow[t]{2}{*}{$\begin{array}{l}\text { Degree of } \\
\text { freedom }\end{array}$} & flavonoids & phenols & carotenoids & $\begin{array}{c}\text { essence } \\
\text { percentage }\end{array}$ & $\begin{array}{l}\text { fresh } \\
\text { flower } \\
\text { matter }\end{array}$ & $\begin{array}{c}\text { Dry } \\
\text { flower } \\
\text { matter }\end{array}$ & $\begin{array}{l}\text { number } \\
\text { of flower }\end{array}$ & $\begin{array}{l}\text { root dry } \\
\text { matter }\end{array}$ & $\begin{array}{l}\text { root } \\
\text { fresh } \\
\text { matter }\end{array}$ & $\begin{array}{c}\text { shoot } \\
\text { dry } \\
\text { matter }\end{array}$ & $\begin{array}{l}\text { shoot } \\
\text { fresh } \\
\text { matter }\end{array}$ \\
\hline & & \multicolumn{11}{|c|}{ Mean of Squares } \\
\hline Year & 1 & 0.067 & 11.1 & 0.009 & 0.002 & 6.7 & 4.4 & 1.1 & 2.2 & 1.2 & 7.6 & 5.2 \\
\hline $\mathrm{R} / \mathrm{Y}$ & 4 & 0.05 & 12 & 0.008 & 0.0032 & 6.6 & 4.7 & 1.2 & 3.3 & 2.3 & 5.5 & 3.3 \\
\hline $\mathrm{Fe}$ & 3 & $0.036^{* *}$ & $95.1^{* *}$ & $0.09^{* *}$ & $0.00004^{\mathrm{ns}}$ & $2.4^{\mathrm{ns}}$ & $2.2^{\mathrm{ns}}$ & $1.8^{*}$ & $4.2^{\circ}$ & $1.5^{\mathrm{ns}}$ & $5.5^{*}$ & $4.1^{*}$ \\
\hline $\mathrm{Zn}$ & 3 & $0.006^{\circ}$ & $49.2^{* *}$ & $0.05^{\circ *}$ & $0.00003^{\mathrm{ns}}$ & $2.5^{\mathrm{ns}}$ & $1.2^{\mathrm{ns}}$ & $1.5^{\mathrm{ns}}$ & $2.4^{\mathrm{ns}}$ & $1.4^{\mathrm{ns}}$ & $4.1^{\mathrm{ns}}$ & $3.9^{\circ}$ \\
\hline $\mathrm{Cu}$ & 3 & $0.005^{\circ}$ & $33.9^{\circ}$ & $0.003^{\circ}$ & $0.00004^{\mathrm{ns}}$ & $2.6^{\mathrm{ns}}$ & $1.9^{\mathrm{ns}}$ & $1.6^{\mathrm{ns}}$ & $2.7^{\mathrm{ns}}$ & $1.5^{\mathrm{ns}}$ & $4.7^{\mathrm{ns}}$ & $2.5^{\mathrm{ns}}$ \\
\hline $\mathrm{Mn}$ & 3 & $0.015^{\circ *}$ & $14.2^{\mathrm{ns}}$ & $0.0031^{\circ}$ & $0.00005^{\mathrm{ns}}$ & $2.4^{\mathrm{ns}}$ & $1.3^{\mathrm{ns}}$ & $1.1^{\mathrm{ns}}$ & $2.5^{\mathrm{ns}}$ & $1.3^{\mathrm{ns}}$ & $5.4^{\mathrm{ns}}$ & $2.7^{\mathrm{ns}}$ \\
\hline $\mathrm{Fe} \times \mathrm{Zn}$ & 9 & $0.004^{\circ}$ & $1421.2^{* *}$ & $0.09^{* *}$ & $0.00008^{\circ}$ & $2.7^{\circ}$ & $3.7^{\circ}$ & $1.2^{\mathrm{ns}}$ & $3.3^{\circ}$ & $1.8^{\circ}$ & $8.6^{\circ *}$ & $3.4^{\circ}$ \\
\hline $\mathrm{Cu} \times \mathrm{Fe}$ & 9 & $0.32^{\circ}$ & $18.4^{\mathrm{ns}}$ & $0.07^{* *}$ & $0.0067^{* *}$ & $6.6^{* *}$ & $3.9^{\circ}$ & $1.3^{\circ}$ & $3.7^{\circ}$ & $3.4^{* *}$ & $4.1^{\circ}$ & $8.8^{* *}$ \\
\hline $\mathrm{Mn} \times \mathrm{Fe}$ & 9 & $0.0038^{*}$ & $23.86^{\circ}$ & $0.0019^{* *}$ & $0.00009^{\circ}$ & $2.8^{\circ}$ & $3.85^{\circ}$ & $2.6^{* *}$ & $7.5^{\circ *}$ & $1.9^{*}$ & $4.2^{*}$ & $8.6^{* *}$ \\
\hline $\mathrm{Cu} \times \mathrm{Zn}$ & 9 & $0.44^{* *}$ & $14.1^{\mathrm{ns}}$ & $0.002^{\circ}$ & $0.012^{* *}$ & $7.8^{* *}$ & $5.8^{* *}$ & $1.4^{\circ}$ & $7.3^{* *}$ & $3.3^{* *}$ & $11.1^{* *}$ & $8.9^{* *}$ \\
\hline $\mathrm{Mn} \times \mathrm{Zn}$ & 9 & $0.1^{* *}$ & $24.1^{\circ}$ & $0.0021^{\circ}$ & $0.000078^{\circ}$ & $2.7^{\circ}$ & $7.6^{* *}$ & $4.7^{* *}$ & $3.4^{\circ}$ & $4.4^{* *}$ & $10.5^{\circ *}$ & $10.3^{* *}$ \\
\hline $\mathrm{Cu} \times \mathrm{Mn}$ & 9 & $0.32^{* *}$ & $55.4^{* \bullet}$ & $0.2^{* *}$ & $0.000081^{\circ}$ & $9.9^{\circ *}$ & $3.6^{\circ}$ & $3.3^{\circ *}$ & $3.3^{\circ}$ & $2.05^{\circ}$ & $11.5^{\circ *}$ & $3.5^{\circ}$ \\
\hline $\mathrm{Cu} \times \mathrm{Fe} \times \mathrm{Zn}$ & 27 & $0.003^{*}$ & $19.1^{*}$ & $0.0015^{*}$ & $0.00007^{*}$ & $2.1^{*}$ & $3.1^{\circ}$ & $1.1^{\circ}$ & $1.9^{\circ}$ & $7.6^{* *}$ & $3.2^{*}$ & $1.87^{\circ}$ \\
\hline $\mathrm{Fe} \times \mathrm{Zn} \times \mathrm{Mn}$ & 27 & $0.22^{* *}$ & $57.6^{* *}$ & $0.7^{* *}$ & $0.069^{* *}$ & $10.6^{* *}$ & $9.7^{* *}$ & $5.4^{* *}$ & $2.1^{\circ}$ & $1.5^{*}$ & $15.5^{* *}$ & $14.4^{* *}$ \\
\hline $\mathrm{Cu} \times \mathrm{Mn} \times \mathrm{Fe}$ & 27 & $0.0034^{\circ}$ & $20.22^{\circ}$ & $0.8^{* *}$ & $0.000075^{\circ}$ & $2.2^{\circ}$ & $9.1^{* *}$ & $1.1^{\circ}$ & $9.8^{* *}$ & $7.4^{* *}$ & $12.5^{\circ *}$ & $14.1^{* *}$ \\
\hline $\mathrm{Cu} \times \mathrm{Mn} \times \mathrm{Zn}$ & 27 & $0.14^{* *}$ & $96.67^{* *}$ & $0.9^{* *}$ & $0.056^{* *}$ & $15.4^{* *}$ & $3.2^{\circ}$ & $1.2^{*}$ & $1.9^{\circ}$ & $1.7^{*}$ & $3.3^{*}$ & $1.8^{\circ}$ \\
\hline $\mathrm{Cu} \times \mathrm{Zn} \times \mathrm{Mn} \times \mathrm{Fe}$ & 81 & $0.0024^{\circ}$ & $15.1^{\circ}$ & $0.0011^{\circ}$ & $0.00006^{\circ}$ & $1.7^{\circ}$ & $2.4^{\circ}$ & $0.9^{\circ}$ & $1.6^{\circ}$ & $1.1^{\circ}$ & $2.5^{\circ}$ & $1.5^{\circ}$ \\
\hline $\mathrm{T}(\mathrm{Cu}, \mathrm{Zn}, \mathrm{Mn}, \mathrm{Fe}) \times \mathrm{Y}$ & 255 & $0.0004^{\circ}$ & $3.1^{\circ}$ & $0.0002^{*}$ & $0.000009^{\circ}$ & $0.3^{\circ}$ & $0.45^{\circ}$ & $0.16^{\circ}$ & $0.28^{\circ}$ & $0.19^{\circ}$ & $0.5^{\circ}$ & $0.3^{\circ}$ \\
\hline E & 1020 & 0.002 & 12.56 & 0.001 & 0.00004 & 1.34 & 1.1 & 0.67 & 1.2 & 0.85 & 2.1 & 1.2 \\
\hline \multicolumn{2}{|c|}{ Coefficient of variation } & 1.1 & 2.2 & 3.3 & 1.2 & 9.7 & 8.7 & 6.3 & 9.1 & 12.1 & 14.4 & 18.3 \\
\hline
\end{tabular}


Table 2- continued Complex analysis of variance of variation of, flavonoids, phenols, carotenoids, essence percentage, fresh flower matter, dry flower matter, number of flowers, and root/shoot dry/fresh matter in plants of Calendula officinalis L.

\begin{tabular}{|c|c|c|c|c|c|c|c|c|c|c|c|c|}
\hline Source of Variation & $\begin{array}{l}\text { Degree of } \\
\text { Freedom }\end{array}$ & flavonoid & phenols & carotenoids & $\begin{array}{c}\text { essence } \\
\text { percentage }\end{array}$ & $\begin{array}{l}\text { flower } \\
\text { fresh } \\
\text { weight }\end{array}$ & $\begin{array}{c}\text { flower } \\
\text { dry } \\
\text { weight }\end{array}$ & $\begin{array}{c}\text { number } \\
\text { of } \\
\text { flowers }\end{array}$ & $\begin{array}{c}\text { root } \\
\text { dry } \\
\text { weight }\end{array}$ & $\begin{array}{l}\text { root } \\
\text { fresh } \\
\text { weight }\end{array}$ & $\begin{array}{l}\text { shoot } \\
\text { dry } \\
\text { weight }\end{array}$ & $\begin{array}{l}\text { shoot } \\
\text { fresh } \\
\text { weight }\end{array}$ \\
\hline & & \multicolumn{11}{|c|}{ Mean of Squares } \\
\hline Year(Y) & 1 & 0.0013 & 0.00002 & 0.00043 & 0.002 & 6.7 & 4.4 & 1.1 & 2.2 & 1.2 & 7.6 & 5.2 \\
\hline $\mathrm{R} / \mathrm{Y}$ & 4 & $0.0035^{\circ}$ & 0.00009 & 0.00032 & 0.0032 & 6.6 & 4.7 & 1.2 & 3.3 & 2.3 & 5.5 & 3.3 \\
\hline Copper $(\mathrm{Cu})$ & 3 & $0.013^{* *}$ & $0.00015^{\circ}$ & $0.0002^{\circ}$ & $0.00004^{\mathrm{ms}}$ & $2.4 \mathrm{~ms}$ & $2.2^{\mathrm{ns}}$ & $1.8^{\circ}$ & $4.2^{\circ}$ & $1.5^{\mathrm{ms}}$ & $5.5^{\mathrm{m}}$ & $4.1^{\circ}$ \\
\hline Manganese (Mn) & 3 & $0.0033^{\circ}$ & $0.00001^{\mathrm{s}}$ & $0.0003^{\circ}$ & $0.00003^{\mathrm{mi}}$ & $2.5^{\mathrm{ms}}$ & $1.2^{\mathrm{ms}}$ & $1.5^{\mathrm{s}}$ & $2.4^{\mathrm{ms}}$ & $1.4^{\mathrm{ms}}$ & $4.1^{\circ}$ & $3.9^{\circ}$ \\
\hline Iron $(\mathrm{Fe})$ & 3 & $0.017^{* *}$ & $0.00007^{\mathrm{ns}}$ & $0.000092^{\mathrm{ms}}$ & $0.00004^{\mathrm{mb}}$ & $2.6^{\mathrm{ms}}$ & $1.9^{\mathrm{ms}}$ & $1.6^{\mathrm{m}}$ & $2.7^{\mathrm{ms}}$ & $1.5^{\mathrm{m}}$ & $4.7^{\circ}$ & $2.5^{\mathrm{s}}$ \\
\hline Boron (B) & 3 & $0.0013^{\mathrm{ms}}$ & $0.00008^{\mathrm{ns}}$ & $0.000001^{\mathrm{ms}}$ & $0.00005^{\mathrm{ms}}$ & $2.4 \mathrm{ss}$ & $1.3^{\mathrm{ns}}$ & $1.1^{\mathrm{m}}$ & $2.5^{\mathrm{ms}}$ & $1.3^{\mathrm{ms}}$ & $5.4^{\mathrm{ms}}$ & $2.7^{\mathrm{ms}}$ \\
\hline $\mathrm{Cu} \times \mathrm{Mn}$ & 9 & $0.0001^{\mathrm{ms}}$ & $0.000083^{\circ}$ & $0.0002^{\circ}$ & $0.00008^{\circ}$ & $2.7^{\circ}$ & $3.7^{\circ}$ & $1.2 \mathrm{ss}$ & $3.3^{\circ}$ & $1.8^{\circ}$ & $8.6^{* *}$ & $3.4^{\circ}$ \\
\hline $\mathrm{Cu} \times \mathrm{Fe}$ & 9 & $0.0001^{m s}$ & $0.000087^{\circ}$ & $0.00023^{\circ}$ & $0.0067^{* *}$ & $6.6^{*}$ & $3.9^{\circ}$ & $1.3^{\circ}$ & $3.7^{\circ}$ & $3.4^{*}$ & $4.1^{\mathrm{ms}}$ & $8.8^{* *}$ \\
\hline $\mathrm{Cu} \times \mathrm{B}$ & 9 & $0.0002^{\circ}$ & $0.0054^{* *}$ & $0.00027^{\circ}$ & $0.00009^{\circ}$ & $2.8^{*}$ & $3.85^{\circ}$ & $2.6^{*}$ & $7.5^{*}$ & $1.9^{\circ}$ & $4.2^{\mathrm{m}}$ & $8.6^{*}$ \\
\hline $\mathrm{Mn} \times \mathrm{Fe}$ & 9 & $0.024^{* *}$ & $0.00009^{\circ}$ & $0.00032^{* *}$ & $0.012^{* *}$ & $7.8^{* *}$ & $5.8^{* *}$ & $1.4^{\circ}$ & $7.3 *$ & $3.3^{* *}$ & $11.1^{* *}$ & $8.9^{* *}$ \\
\hline $\mathrm{Mn} \times \mathrm{B}$ & 9 & $0.00021^{\circ}$ & $0.000082^{\circ}$ & $0.00029^{\circ}$ & $0.000078^{\circ}$ & $2.7^{\circ}$ & $7.6^{*}$ & $4.7^{* *}$ & $3.4^{\circ}$ & 4.4 & 10.5 & $10.3^{* *}$ \\
\hline $\mathrm{Fe} \times \mathrm{B}$ & 9 & $0.003^{* *}$ & $0.000089^{\circ}$ & $0.0003^{\circ}$ & $0.000081^{\circ}$ & $9.9^{* *}$ & $3.6^{\circ}$ & $3.3^{*}$ & $3.3^{\circ}$ & $2.05^{\circ}$ & $11.5^{*}$ & $3.5^{\circ}$ \\
\hline $\mathrm{Cu} \times \mathrm{Mn} \times \mathrm{Fe}$ & 27 & $0.002^{\circ}$ & $0.000075^{\circ}$ & $0.00009^{\circ}$ & $0.00007^{\circ}$ & $2.1^{\circ}$ & $3.1^{\circ}$ & $1.1^{\circ}$ & $1.9^{\circ}$ & $7.6^{*}$ & $3.2^{\circ}$ & $1.87^{\circ}$ \\
\hline $\mathrm{Cu} \times \mathrm{Mn} \times \mathrm{B}$ & 27 & $0.003 *$ & $0.043^{* *}$ & $0.00043^{* *}$ & $0.069^{* *}$ & $10.6^{*}$ & $9.7^{* *}$ & $5.4^{*}$ & $2.1^{\circ}$ & $1.5^{\circ}$ & $15.5^{* *}$ & $14.4^{* *}$ \\
\hline $\mathrm{Cu} \times \mathrm{Fe} \times \mathrm{B}$ & 27 & $0.0025^{\circ}$ & $0.000077^{\circ}$ & $0.000095^{\circ}$ & $0.000075^{\circ}$ & $2.2^{\circ}$ & $9.1^{* *}$ & $1.1^{\circ}$ & $9.8^{* *}$ & $7.4^{*}$ & $12.5^{* *}$ & $14.1^{* *}$ \\
\hline $\mathrm{Fe} \times \mathrm{B} \times \mathrm{Mn}$ & 27 & $0.045^{* *}$ & $0.024^{* *}$ & $0.0045^{* *}$ & $0.056^{* *}$ & $15.4^{* *}$ & $3.2^{\circ}$ & $1.2^{\circ}$ & $1.9^{\circ}$ & $1.7^{\circ}$ & $3.3^{\circ}$ & $1.8^{\circ}$ \\
\hline $\mathrm{Cu} \times \mathrm{B} \times \mathrm{Mn} \times \mathrm{Fe}$ & 81 & $0.002^{\circ}$ & $0.000065^{\circ}$ & $0.00007^{\circ}$ & $0.00006^{\circ}$ & $1.7^{\circ}$ & $2.4^{\circ}$ & $0.9^{\circ}$ & $1.6^{\circ}$ & $1.1^{\circ}$ & $2.5^{\circ}$ & $1.5^{\circ}$ \\
\hline $\mathrm{T}(\mathrm{Cu}, \mathrm{B}, \mathrm{Mn}, \mathrm{Fe}) \times \mathrm{Y}$ & 255 & $0.0002^{\circ}$ & $0.0000093^{\circ}$ & $0.000019^{\circ}$ & $0.000009^{\circ}$ & $0.3^{\circ}$ & $0.45^{*}$ & $0.16^{\circ}$ & $0.28^{\circ}$ & $0.19^{\circ}$ & $0.5^{\circ}$ & $0.3^{\circ}$ \\
\hline $\mathrm{E}$ & 1020 & 0.0009 & 0.00004 & 0.000054 & 0.00004 & 1.34 & 1.1 & 0.67 & 1.2 & 0.85 & 2.1 & 1.2 \\
\hline $\mathrm{CV}$ & & 1.1 & 2.4 & 3.3 & 1.2 & 9.7 & 8.7 & 6.8 & 9.2 & 16.9 & 14.2 & 19.4 \\
\hline
\end{tabular}

Table 2. continued Complex analysis of variance of variation of, Carvacrol percentage, Thymol percentage, essence percentage, number of stem, height,and root/shoot dry/fresh matter in plants of Thymus vulgaris L.

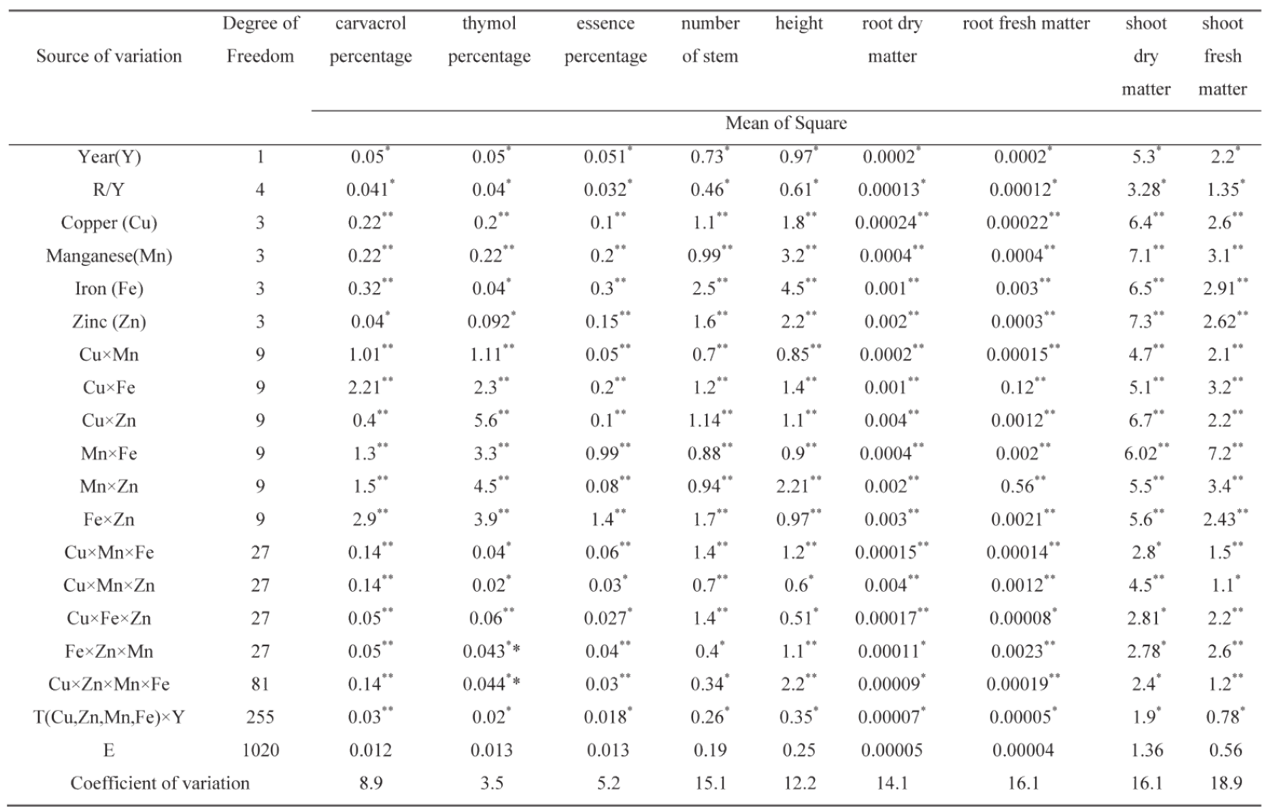


Table 3. Results of correlation between characters in borago plants that are affected by several micronutrients (2012).

\begin{tabular}{|c|c|c|c|c|c|c|c|c|c|c|c|}
\hline Characters & $\begin{array}{c}\text { shoot } \\
\text { fresh } \\
\text { weight } \\
\text { (1) }\end{array}$ & $\begin{array}{c}\text { shoot } \\
\text { dry } \\
\text { weight } \\
\text { (2) }\end{array}$ & $\begin{array}{c}\begin{array}{c}\text { root } \\
\text { fresh }\end{array} \\
\text { weight } \\
\text { (3) }\end{array}$ & 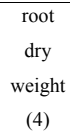 & $\begin{array}{c}\text { number } \\
\text { of } \\
\text { flowers } \\
(5)\end{array}$ & $\begin{array}{c}\text { flower } \\
\text { dry } \\
\text { weight } \\
(6)\end{array}$ & $\begin{array}{l}\text { flower } \\
\text { fresh } \\
\text { weight } \\
\text { (7) }\end{array}$ & $\begin{array}{c}\text { Extraction } \\
\text { percentage } \\
\text { (8) }\end{array}$ & $\begin{array}{c}\text { Carotenoid } \\
\text { (9) }\end{array}$ & $\begin{array}{c}\text { Phenol } \\
(10)\end{array}$ & $\begin{array}{c}\text { Flavonoid } \\
\text { (11) }\end{array}$ \\
\hline 1 & 1 & & & & & & & & & & \\
\hline 2 & $0.52^{*}$ & 1 & & & & & & & & & \\
\hline 3 & 0.26 & 0.1 & 1 & & & & & & & & \\
\hline 4 & 0.36 & $0.56^{* *}$ & $0.66^{* *}$ & 1 & & & & & & & \\
\hline 5 & $0.5^{*}$ & $0.58^{* *}$ & $0.64^{* *}$ & $0.52^{*}$ & 1 & & & & & & \\
\hline 6 & $0.5^{*}$ & $0.76^{* *}$ & $0.5^{*}$ & 0.44 & $0.58^{* *}$ & 1 & & & & & \\
\hline 7 & $0.6^{* *}$ & $0.59^{* *}$ & 0.35 & 0.36 & $0.55^{* *}$ & $0.58^{* *}$ & 1 & & & & \\
\hline 8 & 0.48 & $0.56^{* *}$ & 0.46 & 0.33 & $0.7^{* *}$ & $0.65^{* *}$ & 0.46 & 1 & & & \\
\hline 9 & 0.1 & $0.68^{* *}$ & 0.11 & 0.4 & 0.4 & $0.7^{* *}$ & 0.26 & $0.69^{* *}$ & 1 & & \\
\hline 10 & 0.11 & $0.76^{* *}$ & 0.1 & 0.3 & 0.42 & 0.4 & 0.24 & $0.66^{* *}$ & 0.48 & 1 & \\
\hline 11 & 0.1 & $0.56^{* *}$ & 0.3 & $0.55^{*}$ & 0.31 & 0.42 & 0.33 & $0.68^{* *}$ & 0.26 & 0.36 & 1 \\
\hline
\end{tabular}

Table 3. continued Results of correlation between characters in borago plants that are affected by several micronutrients (2013).

\begin{tabular}{|c|c|c|c|c|c|c|c|c|c|c|c|}
\hline Characters & $\begin{array}{c}\text { shoot } \\
\text { fresh } \\
\text { weight } \\
\text { (1) }\end{array}$ & $\begin{array}{c}\text { shoot } \\
\text { dry } \\
\text { weight } \\
\text { (2) }\end{array}$ & $\begin{array}{c}\text { root } \\
\text { fresh } \\
\text { weight } \\
\text { (3) }\end{array}$ & $\begin{array}{c}\text { root dry } \\
\text { weight } \\
\text { (4) }\end{array}$ & $\begin{array}{c}\text { number } \\
\text { of } \\
\text { flowers } \\
(5)\end{array}$ & $\begin{array}{c}\text { flower } \\
\text { dry } \\
\text { weight } \\
(6)\end{array}$ & $\begin{array}{c}\text { flower } \\
\text { fresh } \\
\text { weight } \\
\text { (7) }\end{array}$ & $\begin{array}{c}\text { essence } \\
\text { percentage } \\
\text { (8) }\end{array}$ & $\begin{array}{c}\text { Carotenoid } \\
\text { (9) }\end{array}$ & $\begin{array}{c}\text { Phenol } \\
(10)\end{array}$ & $\begin{array}{c}\text { Flavonoid } \\
\text { (11) }\end{array}$ \\
\hline 1 & 1 & & & & & & & & & & \\
\hline 2 & $0.56^{* *}$ & 1 & & & & & & & & & \\
\hline 3 & 0.36 & 0.2 & 1 & & & & & & & & \\
\hline 4 & 0.36 & $0.66^{* *}$ & $0.73^{* *}$ & 1 & & & & & & & \\
\hline 5 & $0.5^{*}$ & $0.68^{* *}$ & $0.65^{* *}$ & $0.7^{* * *}$ & 1 & & & & & & \\
\hline 6 & 0.48 & $0.8^{* *}$ & $0.6^{* *}$ & 0.4 & $0.8^{* *}$ & 1 & & & & & \\
\hline 7 & $0.78^{* *}$ & $0.7^{* *}$ & 0.3 & 0.3 & $0.87^{* *}$ & $0.87^{* *}$ & 1 & & & & \\
\hline 8 & $0.58^{*}$ & $0.6^{* *}$ & 0.4 & 0.3 & $0.7^{* *}$ & $0.7^{* *}$ & $0.6^{* *}$ & 1 & & & \\
\hline 9 & 0.2 & $0.6^{* *}$ & 0.14 & 0.4 & 0.3 & $0.8^{* *}$ & 0.3 & $0.8^{* *}$ & 1 & & \\
\hline 10 & 0.22 & $0.8^{* *}$ & 0.2 & 0.3 & 0.4 & 0.3 & 0.4 & $0.5^{*}$ & 0.3 & 1 & \\
\hline 11 & 0.3 & $0.7^{* *}$ & 0.2 & $0.5^{*}$ & 0.3 & 0.4 & 0.2 & 0.48 & 0.3 & 0.4 & 1 \\
\hline
\end{tabular}


Table 3 - continued Results of correlation between characters in Calendula plants that are affected by several micronutrients (2012)

\begin{tabular}{|c|c|c|c|c|c|c|c|c|c|c|c|}
\hline Characters & $\begin{array}{c}\text { shoot } \\
\text { fresh } \\
\text { weight } \\
\text { (1) }\end{array}$ & $\begin{array}{l}\text { shoot } \\
\text { dry } \\
\text { weight } \\
\text { (2) }\end{array}$ & $\begin{array}{c}\text { root } \\
\text { fresh } \\
\text { weight } \\
\text { (3) }\end{array}$ & $\begin{array}{c}\text { root } \\
\text { dry } \\
\text { weight } \\
\text { (4) }\end{array}$ & $\begin{array}{c}\text { number } \\
\text { of } \\
\text { flowers } \\
(5)\end{array}$ & $\begin{array}{c}\text { flower } \\
\text { dry } \\
\text { weight } \\
(6)\end{array}$ & $\begin{array}{c}\text { flower } \\
\text { fresh } \\
\text { weight } \\
\text { (7) }\end{array}$ & $\begin{array}{c}\text { essence } \\
\text { percentage } \\
\text { (8) }\end{array}$ & $\begin{array}{c}\text { Carotenoid } \\
\text { (9) }\end{array}$ & $\begin{array}{c}\text { Phenol } \\
(10)\end{array}$ & $\begin{array}{c}\text { Flavonoid } \\
\text { (11) }\end{array}$ \\
\hline 1 & 1 & & & & & & & & & & \\
\hline 2 & $0.9^{* *}$ & 1 & & & & & & & & & \\
\hline 3 & $0.89^{* *}$ & $0.91^{* *}$ & 1 & & & & & & & & \\
\hline 4 & $0.85^{* *}$ & $0.9^{* *}$ & 0.34 & 1 & & & & & & & \\
\hline 5 & $0.76^{* *}$ & $0.87^{* * *}$ & $0.9^{* * *}$ & $0.69^{* *}$ & 1 & & & & & & \\
\hline 6 & $0.73^{* * *}$ & $0.78^{* * *}$ & $0.9^{* * *}$ & $0.59^{* * *}$ & $0.9^{* * *}$ & 1 & & & & & \\
\hline 7 & $0.95^{* *}$ & $0.96^{* *}$ & $0.9^{* *}$ & $0.54^{* *}$ & $0.77^{* *}$ & $0.79^{* *}$ & 1 & & & & \\
\hline 8 & $0.94^{* *}$ & $0.95^{* *}$ & 0.44 & 0.42 & $0.8^{* *}$ & $0.7^{* *}$ & $0.68^{* *}$ & 1 & & & \\
\hline 9 & $0.82^{* *}$ & $0.94^{* *}$ & 0.42 & 0.37 & $0.8^{* *}$ & $0.68^{* *}$ & $0.78^{* *}$ & 0.5 & 1 & & \\
\hline 10 & $0.92^{* *}$ & $0.94^{* *}$ & 0.37 & 0.36 & $0.6^{* *}$ & $0.8^{* *}$ & $0.76^{* *}$ & 0.46 & 0.5 & 1 & \\
\hline 11 & $0.87^{* *}$ & $0.92^{* *}$ & $0.6^{* *}$ & $0.55^{* *}$ & $0.65^{* *}$ & $0.56^{* *}$ & $0.6^{* *}$ & $0.69^{* * *}$ & $0.69^{* * *}$ & $0.65^{* *}$ & 1 \\
\hline
\end{tabular}

Table 3 . continued Results of correlation between characters in Calendula plants that are affected by several micronutrients (2013).

\begin{tabular}{|c|c|c|c|c|c|c|c|c|c|c|c|}
\hline Characters & $\begin{array}{c}\text { shoot } \\
\text { fresh } \\
\text { weight } \\
\text { (1) }\end{array}$ & $\begin{array}{c}\text { shoot } \\
\text { dry } \\
\text { weight } \\
(2)\end{array}$ & $\begin{array}{c}\text { root } \\
\text { fresh } \\
\text { weight } \\
\text { (3) }\end{array}$ & $\begin{array}{c}\text { root } \\
\text { dry } \\
\text { weight } \\
\text { (4) }\end{array}$ & $\begin{array}{c}\text { number } \\
\text { of } \\
\text { flowers } \\
(5)\end{array}$ & $\begin{array}{c}\text { flower } \\
\text { dry } \\
\text { weight } \\
(6)\end{array}$ & 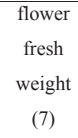 & $\begin{array}{c}\text { essence } \\
\text { percentage } \\
(8)\end{array}$ & $\begin{array}{c}\text { Carotenoid } \\
\text { (9) }\end{array}$ & $\begin{array}{c}\text { Phenol } \\
(10)\end{array}$ & $\begin{array}{c}\text { Flavonoid } \\
\text { (11) }\end{array}$ \\
\hline 1 & 1 & & & & & & & & & & \\
\hline 2 & $0.91^{* *}$ & 1 & & & & & & & & & \\
\hline 3 & $0.99^{* *}$ & $0.87^{* *}$ & 1 & & & & & & & & \\
\hline 4 & $0.8^{* *}$ & $0.82^{* *}$ & 0.34 & 1 & & & & & & & \\
\hline 5 & $0.7^{* *}$ & $0.8^{* *}$ & $0.89^{* *}$ & $0.89^{* *}$ & 1 & & & & & & \\
\hline 6 & $0.7^{* *}$ & $0.88^{* *}$ & $0.69^{* *}$ & $0.59^{* *}$ & $0.79^{* *}$ & 1 & & & & & \\
\hline 7 & $0.9^{* *}$ & $0.86^{* *}$ & $0.79^{* *}$ & $0.74^{* *}$ & $0.82^{* *}$ & $0.89^{* *}$ & 1 & & & & \\
\hline 8 & $0.92^{* *}$ & $0.9^{* *}$ & 0.34 & 0.42 & $0.68^{* *}$ & $0.87^{* *}$ & $0.88^{* *}$ & 1 & & & \\
\hline 9 & $0.8^{* *}$ & $0.9^{* *}$ & 0.22 & 0.27 & $0.78^{* *}$ & $0.88^{* *}$ & $0.88^{* *}$ & 0.5 & 1 & & \\
\hline 10 & $0.9^{* *}$ & $0.9^{* *}$ & 0.37 & 0.26 & $0.6^{* *}$ & $0.78^{* *}$ & $0.86^{* *}$ & $0.55^{*}$ & 0.5 & 1 & \\
\hline 11 & $0.8^{* *}$ & $0.9^{* *}$ & $0.76^{* *}$ & $0.65^{* *}$ & $0.65^{* *}$ & $0.76^{* *}$ & $0.96^{* *}$ & $0.9^{* *}$ & $0.59^{*}$ & $0.6^{* *}$ & 1 \\
\hline
\end{tabular}


Table 3. continued Results of correlation between characters in Thyme plants that are affected by several micronutrients (2012).

\begin{tabular}{|c|c|c|c|c|c|c|c|c|c|}
\hline Characters & 1 & 2 & 3 & 4 & 5 & 6 & 7 & 8 & 9 \\
\hline Shoot dry matter (1) & 1 & & & & & & & & \\
\hline Shoot fresh matter (2) & $0.92^{* *}$ & 1 & & & & & & & \\
\hline Root dry matter (3) & $0.99^{* *}$ & $0.9^{* *}$ & 1 & & & & & & \\
\hline Root fresh matter (4) & $0.75^{* *}$ & $0.65^{* *}$ & $0.66^{* *}$ & 1 & & & & & \\
\hline Height (5) & $0.66^{* *}$ & $0.6^{* *}$ & $0.66^{* *}$ & $0.6^{* *}$ & 1 & & & & \\
\hline Number of stems (6) & $0.77^{* *}$ & $0.57^{* *}$ & $0.67^{* *}$ & $0.57^{* *}$ & $0.87^{* *}$ & 1 & & & \\
\hline Essence percentage (7) & $0.94^{* *}$ & $0.74^{* *}$ & $0.79^{* *}$ & $0.7^{* *}$ & $0.7^{* *}$ & $0.7^{* *}$ & 1 & & \\
\hline Carvacrol percentage (8) & $0.91^{* *}$ & $0.81^{* *}$ & $0.85^{* *}$ & $0.8^{* *}$ & $0.92^{* *}$ & $0.94^{* *}$ & $0.98^{* *}$ & 1 & \\
\hline Thymol percentage (9) & $0.84^{* *}$ & $0.8^{* *}$ & $0.84^{* *}$ & $0.72^{* *}$ & $0.82^{* *}$ & $0.92^{* *}$ & $0.97^{* *}$ & $0.78^{* *}$ & 1 \\
\hline
\end{tabular}

Table 3. continued Results of correlation between characters in Thyme plants that are affected by several micronutrients (2013).

\begin{tabular}{|c|c|c|c|c|c|c|c|c|c|}
\hline Characters & 1 & 2 & 3 & 4 & 5 & 6 & 7 & 8 & 9 \\
\hline Shoot dry matter (1) & 1 & & & & & & & & \\
\hline Shoot fresh matter (2) & $0.9^{* *}$ & 1 & & & & & & & \\
\hline Root dry matter (3) & $0.89^{* *}$ & $0.78^{* *}$ & 1 & & & & & & \\
\hline Root fresh matter (4) & $0.85^{*}$ & $0.62^{* *}$ & $0.64^{* *}$ & 1 & & & & & \\
\hline Height (5) & $0.76^{*}$ & $0.57^{* *}$ & $0.63^{* *}$ & $0.76^{*}$ & 1 & & & & \\
\hline Number of stems (6) & $0.73^{* *}$ & $0.55^{* *}$ & $0.62^{* *}$ & $0.67^{* *}$ & $0.97^{*}$ & 1 & & & \\
\hline Essence percentage (7) & $0.95^{*}$ & $0.73^{*}$ & $0.89^{* *}$ & $0.67^{* *}$ & $0.77^{*}$ & $0.77^{* *}$ & 1 & & \\
\hline Carvacrol percentage (8) & $0.94^{* *}$ & $0.8^{* *}$ & $0.87^{* *}$ & $0.88^{* *}$ & $0.9^{* *}$ & $0.9^{* *}$ & $0.9^{* *}$ & 1 & \\
\hline Thymol percentage (9) & $0.82^{* *}$ & $0.76^{* *}$ & $0.85^{* *}$ & $0.78^{* *}$ & $0.87^{* *}$ & $0.96^{*}$ & $0.95^{* *}$ & $0.88^{* *}$ & 1 \\
\hline
\end{tabular}

ns: Not Significant, *and** : Significant at $P=0.05$ and $P=0.01$ levels of probability, respectively.

Table 4. Means of characters measured in borago plants that are affected by micronutrients ( $400 \mathrm{ppm})$ concentration and control plants ( $1^{\text {styear}) . ~}$

\begin{tabular}{|c|c|c|c|c|c|c|c|c|c|c|c|}
\hline Treatments & flavonoids & phenols & carotenoids & $\begin{array}{c}\text { essence } \\
\text { percentage }\end{array}$ & $\begin{array}{c}\text { fresh } \\
\text { flower } \\
\text { matter }\end{array}$ & $\begin{array}{c}\text { Dry } \\
\text { flower } \\
\text { matter }\end{array}$ & $\begin{array}{c}\text { number } \\
\text { of } \\
\text { flower }\end{array}$ & $\begin{array}{c}\text { root } \\
\text { dry } \\
\text { matter }\end{array}$ & $\begin{array}{c}\text { root } \\
\text { fresh } \\
\text { matter }\end{array}$ & $\begin{array}{c}\text { shoot } \\
\text { dry } \\
\text { matter }\end{array}$ & $\begin{array}{l}\text { shoot } \\
\text { fresh } \\
\text { matter }\end{array}$ \\
\hline $\mathrm{Fe}$ & $0.03 \mathrm{~cd}$ & $12.1 \mathrm{c}$ & $0.07 \mathrm{de}$ & $0.24 \mathrm{~d}$ & $13 \mathrm{c}$ & $6.8 \mathrm{~cd}$ & $7 \mathrm{~cd}$ & $15 \mathrm{~cd}$ & $21.3 \mathrm{de}$ & $16.7 \mathrm{c}$ & $32.3 \mathrm{~d}$ \\
\hline $\mathrm{Zn}$ & $0.04 \mathrm{~cd}$ & $12.2 \mathrm{c}$ & $0.11 d$ & $0.22 \mathrm{~d}$ & $10.7 \mathrm{~cd}$ & $6.5 \mathrm{~cd}$ & $8 \mathrm{c}$ & $12.7 \mathrm{~cd}$ & $23.3 \mathrm{de}$ & $15.7 \mathrm{c}$ & $32.6 \mathrm{~cd}$ \\
\hline $\mathrm{Cu}$ & $0.07 \mathrm{c}$ & $12.4 \mathrm{c}$ & $0.095 \mathrm{~d}$ & $0.25 \mathrm{~d}$ & $13 \mathrm{c}$ & $6.3 \mathrm{~cd}$ & $8.1 \mathrm{c}$ & $12.5 \mathrm{~cd}$ & $22.3 \mathrm{de}$ & $17.4 \mathrm{bc}$ & $32.8 \mathrm{~cd}$ \\
\hline $\mathrm{Mn}$ & $0.09 \mathrm{c}$ & $13.1 \mathrm{c}$ & $0.1 \mathrm{~d}$ & $0.24 \mathrm{~d}$ & $10.7 \mathrm{~cd}$ & $6.5 \mathrm{~cd}$ & $6.7 \mathrm{~d}$ & $12.5 \mathrm{~cd}$ & $20.4 \mathrm{de}$ & $16.2 \mathrm{c}$ & $32.3 \mathrm{~d}$ \\
\hline $\mathrm{Fe} \times \mathrm{Zn}$ & $0.11 \mathrm{c}$ & $13.5 \mathrm{c}$ & $0.23 \mathrm{bc}$ & $0.56 \mathrm{c}$ & $11.6 \mathrm{~cd}$ & $7.4 \mathrm{~cd}$ & $9.2 \mathrm{~b}$ & $14.6 \mathrm{~cd}$ & $24.8 \mathrm{~d}$ & $17.3 \mathrm{bc}$ & $33.3 \mathrm{~cd}$ \\
\hline $\mathrm{Cu} \times \mathrm{Fe}$ & $0.1 \mathrm{c}$ & $19 \mathrm{~cd}$ & $0.22 \mathrm{bc}$ & $0.52 \mathrm{c}$ & $13 \mathrm{c}$ & $7.7 \mathrm{~cd}$ & $9.3 \mathrm{~b}$ & $16 \mathrm{~cd}$ & $26.3 \mathrm{~cd}$ & $17.5 \mathrm{bc}$ & $33.8 \mathrm{~cd}$ \\
\hline $\mathrm{Mn} \times \mathrm{Fe}$ & $0.28 \mathrm{~b}$ & $20 \mathrm{~cd}$ & $0.18 b c$ & $0.55 \mathrm{c}$ & $13 \mathrm{c}$ & $8.8 \mathrm{c}$ & $9.5 \mathrm{~b}$ & $18 \mathrm{c}$ & $28.1 \mathrm{c}$ & $17.9 \mathrm{bc}$ & $34.2 \mathrm{~cd}$ \\
\hline $\mathrm{Cu} \times \mathrm{Zn}$ & $0.29 \mathrm{~b}$ & $25 \mathrm{c}$ & $0.17 b c$ & $0.59 \mathrm{bc}$ & $12 \mathrm{~cd}$ & $8.5 \mathrm{~cd}$ & $9.6 \mathrm{~b}$ & $16.7 \mathrm{~cd}$ & $27.3 \mathrm{~cd}$ & $19.2 \mathrm{bc}$ & $35.2 \mathrm{c}$ \\
\hline $\mathrm{Mn} \times \mathrm{Zn}$ & $0.31 \mathrm{~b}$ & $22 \mathrm{c}$ & $0.15 \mathrm{c}$ & $0.54 \mathrm{c}$ & $13 \mathrm{c}$ & $8.1 \mathrm{~cd}$ & $9.6 \mathrm{~b}$ & $16.4 \mathrm{~cd}$ & $28.3 \mathrm{c}$ & $16.6 \mathrm{c}$ & $34.3 \mathrm{~cd}$ \\
\hline $\mathrm{Cu} \times \mathrm{Mn}$ & $0.16 b c$ & $35 \mathrm{bc}$ & $0.25 b c$ & $0.62 b c$ & $13 \mathrm{c}$ & $8.2 \mathrm{~cd}$ & $9.7 \mathrm{ab}$ & $16.2 \mathrm{~cd}$ & $27.4 \mathrm{~cd}$ & $17.5 \mathrm{bc}$ & $36.7 \mathrm{bc}$ \\
\hline $\mathrm{Cu} \times \mathrm{Fe} \times \mathrm{Zn}$ & $0.15 b c$ & $49 b$ & $0.3 b$ & $0.71 \mathrm{~b}$ & $15 \mathrm{~b}$ & $9.8 \mathrm{bc}$ & $10.3 \mathrm{a}$ & $20 \mathrm{bc}$ & $31.3 \mathrm{bc}$ & $22 \mathrm{ab}$ & $36.8 \mathrm{bc}$ \\
\hline $\mathrm{Fe} \times \mathrm{Zn} \times \mathrm{Mn}$ & $0.3 b$ & $50 \mathrm{~b}$ & $0.38 \mathrm{~b}$ & $0.7 \mathrm{~b}$ & $17 \mathrm{ab}$ & $9.9 \mathrm{bc}$ & $10.5 \mathrm{a}$ & $20 \mathrm{bc}$ & $32.5 \mathrm{bc}$ & $21.3 \mathrm{ab}$ & $37.2 \mathrm{bc}$ \\
\hline $\mathrm{Cu} \times \mathrm{Mn} \times \mathrm{Fe}$ & $0.43 \mathrm{ab}$ & $65 \mathrm{ab}$ & $0.39 \mathrm{~b}$ & $0.8 \mathrm{ab}$ & $15 \mathrm{~b}$ & $10.8 \mathrm{~b}$ & $10.5 \mathrm{a}$ & $22 b$ & $34.3 \mathrm{~b}$ & $22.7 \mathrm{ab}$ & $37.5 \mathrm{bc}$ \\
\hline $\mathrm{Cu} \times \mathrm{Mn} \times \mathrm{Zn}$ & $0.45 \mathrm{ab}$ & $55 \mathrm{~b}$ & $0.4 \mathrm{~b}$ & $0.8 \mathrm{ab}$ & $15.3 \mathrm{~b}$ & $11.1 \mathrm{~b}$ & $10.2 \mathrm{a}$ & $22.3 \mathrm{~b}$ & $34.9 \mathrm{~b}$ & $20.2 b$ & $38.2 \mathrm{~b}$ \\
\hline $\mathrm{Cu} \times \mathrm{Zn} \times \mathrm{Mn} \times \mathrm{Fe}$ & $0.6 \mathrm{a}$ & $75 \mathrm{a}$ & $0.6 \mathrm{a}$ & $0.9 \mathrm{a}$ & $19 \mathrm{a}$ & $14.8 \mathrm{a}$ & $10.9 \mathrm{a}$ & $34.1 \mathrm{a}$ & $44.8 \mathrm{a}$ & $30.9 \mathrm{a}$ & $42.2 \mathrm{a}$ \\
\hline Control & $0.01 \mathrm{~d}$ & $8 \mathrm{~d}$ & $0.05 \mathrm{e}$ & $0.2 \mathrm{~d}$ & $10 \mathrm{~d}$ & $5.5 \mathrm{~d}$ & $7 \mathrm{~d}$ & $12 \mathrm{~d}$ & $20.1 \mathrm{e}$ & $10.3 \mathrm{~d}$ & $20.4 \mathrm{e}$ \\
\hline
\end{tabular}


Table 4b. Means of Characters measured in borago plants that are affected by micronutrients (400 ppm) concentration and control plants ( $2^{\text {nd }}$ year $)$.

\begin{tabular}{|c|c|c|c|c|c|c|c|c|c|c|c|}
\hline Treatments & flavonoids & phenols & carotenoids & $\begin{array}{c}\text { essence } \\
\text { percentage }\end{array}$ & $\begin{array}{c}\text { fresh } \\
\text { flower } \\
\text { matter }\end{array}$ & $\begin{array}{c}\text { Dry } \\
\text { flower } \\
\text { matter }\end{array}$ & $\begin{array}{c}\text { number } \\
\text { of } \\
\text { flower }\end{array}$ & $\begin{array}{c}\text { root } \\
\text { dry } \\
\text { matter }\end{array}$ & $\begin{array}{c}\text { root } \\
\text { fresh } \\
\text { matter }\end{array}$ & $\begin{array}{l}\text { shoot } \\
\text { dry } \\
\text { matter }\end{array}$ & $\begin{array}{l}\text { shoot } \\
\text { fresh } \\
\text { matter }\end{array}$ \\
\hline $\mathrm{Fe}$ & $0.08 \mathrm{c}$ & $14 \mathrm{c}$ & $0.07 \mathrm{de}$ & $0.23 \mathrm{~d}$ & $15 \mathrm{c}$ & $7.5 \mathrm{~d}$ & $10 \mathrm{~cd}$ & $16.2 \mathrm{~cd}$ & $32.5 \mathrm{~d}$ & $15.7 \mathrm{c}$ & $39.3 \mathrm{~d}$ \\
\hline $\mathrm{Zn}$ & $0.05 \mathrm{~cd}$ & $12 \mathrm{c}$ & $0.11 \mathrm{~d}$ & $0.21 \mathrm{~d}$ & $15 \mathrm{c}$ & $8.5 \mathrm{~cd}$ & $11 \mathrm{c}$ & $14.9 \mathrm{~cd}$ & $32.5 \mathrm{~d}$ & $12.7 \mathrm{c}$ & $39.6 \mathrm{~cd}$ \\
\hline $\mathrm{Cu}$ & $0.06 \mathrm{c}$ & $11 \mathrm{c}$ & $0.095 \mathrm{~d}$ & $0.5 \mathrm{c}$ & $15 \mathrm{c}$ & $7.7 \mathrm{~d}$ & $11.1 \mathrm{c}$ & $14.7 \mathrm{~cd}$ & $35.7 \mathrm{~d}$ & $18.4 \mathrm{bc}$ & $36.3 \mathrm{~d}$ \\
\hline $\mathrm{Mn}$ & $0.03 \mathrm{~cd}$ & $11.1 \mathrm{c}$ & $0.07 \mathrm{de}$ & $0.24 \mathrm{~d}$ & $11 \mathrm{~d}$ & $8.5 \mathrm{~cd}$ & $8.7 \mathrm{~d}$ & $13 \mathrm{~d}$ & $30.1 \mathrm{e}$ & $15.4 \mathrm{c}$ & $30.2 \mathrm{de}$ \\
\hline $\mathrm{Fe} \times \mathrm{Zn}$ & $0.09 \mathrm{c}$ & $11.5 \mathrm{c}$ & $0.23 \mathrm{bc}$ & $0.5 \mathrm{c}$ & $13.6 \mathrm{~cd}$ & $9.4 \mathrm{~cd}$ & $11.2 \mathrm{c}$ & $14.8 \mathrm{~d}$ & $30.1 \mathrm{e}$ & $18.4 \mathrm{bc}$ & $42.5 \mathrm{~cd}$ \\
\hline $\mathrm{Cu} \times \mathrm{Fe}$ & $0.09 \mathrm{c}$ & $21 \mathrm{~cd}$ & $0.22 b c$ & $0.5 \mathrm{c}$ & $13.4 \mathrm{~cd}$ & $9.7 \mathrm{~cd}$ & $12.3 \mathrm{~b}$ & $20.5 \mathrm{c}$ & $35.3 \mathrm{c}$ & $18.5 \mathrm{bc}$ & $42.5 \mathrm{~cd}$ \\
\hline $\mathrm{Mn} \times \mathrm{Fe}$ & $0.25 b$ & $22 \mathrm{~cd}$ & $0.2 \mathrm{c}$ & $0.5 \mathrm{c}$ & $15 \mathrm{c}$ & $10.8 \mathrm{c}$ & $11.5 \mathrm{c}$ & $20.5 \mathrm{c}$ & $40.2 \mathrm{c}$ & $18.9 \mathrm{bc}$ & $45.2 \mathrm{c}$ \\
\hline $\mathrm{Cu} \times \mathrm{Zn}$ & $0.15 b c$ & $30 \mathrm{c}$ & $0.2 \mathrm{c}$ & $0.61 b c$ & $14 \mathrm{~cd}$ & $10.5 \mathrm{~cd}$ & $12.6 \mathrm{~b}$ & $20 \mathrm{c}$ & $41.3 \mathrm{c}$ & $21 b c$ & $45.2 \mathrm{c}$ \\
\hline $\mathrm{Mn} \times \mathrm{Zn}$ & $0.12 b c$ & $31 \mathrm{c}$ & $0.2 \mathrm{c}$ & $0.54 \mathrm{c}$ & $15 \mathrm{c}$ & $10.9 \mathrm{c}$ & $12.6 \mathrm{~b}$ & $18.6 \mathrm{~cd}$ & $42.3 \mathrm{c}$ & $15.6 \mathrm{c}$ & $45.2 \mathrm{c}$ \\
\hline $\mathrm{Cu} \times \mathrm{Mn}$ & $0.11 b c$ & $35 \mathrm{bc}$ & $0.35 b c$ & $0.5 \mathrm{c}$ & $15 \mathrm{c}$ & $10.2 \mathrm{~cd}$ & $12.7 \mathrm{ab}$ & $18.4 \mathrm{~cd}$ & $44.4 \mathrm{c}$ & $18.5 b c$ & $51.7 \mathrm{bc}$ \\
\hline $\mathrm{Cu} \times \mathrm{Fe} \times \mathrm{Zn}$ & $0.3 b$ & $50 \mathrm{~b}$ & $0.35 b c$ & $0.75 b$ & $17 \mathrm{~b}$ & $11.8 \mathrm{bc}$ & $13.3 \mathrm{a}$ & $22 \mathrm{bc}$ & $52.3 \mathrm{bc}$ & $24 \mathrm{ab}$ & $51.8 \mathrm{bc}$ \\
\hline $\mathrm{Fe} \times \mathrm{Zn} \times \mathrm{Mn}$ & $0.4 \mathrm{~b}$ & $38 \mathrm{bc}$ & $0.4 \mathrm{~b}$ & $0.72 b$ & $19 \mathrm{ab}$ & $13 \mathrm{~b}$ & $12.5 \mathrm{~b}$ & $23 \mathrm{bc}$ & $54.5 \mathrm{bc}$ & $24.3 \mathrm{ab}$ & $54.2 \mathrm{~b}$ \\
\hline $\mathrm{Cu} \times \mathrm{Mn} \times \mathrm{Fe}$ & $0.48 b$ & $72 \mathrm{a}$ & $0.4 \mathrm{~b}$ & $0.9 \mathrm{ab}$ & $17 \mathrm{~b}$ & $12.8 \mathrm{~b}$ & $12.5 \mathrm{~b}$ & $28 \mathrm{~b}$ & $56.3 \mathrm{~b}$ & $24.7 \mathrm{ab}$ & $54.5 \mathrm{~b}$ \\
\hline $\mathrm{Cu} \times \mathrm{Mn} \times \mathrm{Zn}$ & $0.34 \mathrm{~b}$ & $68 \mathrm{a}$ & $0.41 \mathrm{~b}$ & $0.72 b$ & $17.5 \mathrm{~b}$ & $13.1 \mathrm{~b}$ & $13.2 \mathrm{a}$ & $24.5 b$ & $57.9 \mathrm{~b}$ & $30.2 \mathrm{~b}$ & $54.2 \mathrm{~b}$ \\
\hline $\mathrm{Cu} \times \mathrm{Zn} \times \mathrm{Mn} \times \mathrm{Fe}$ & $0.62 \mathrm{a}$ & $75 \mathrm{a}$ & $0.8 \mathrm{a}$ & $1.1 \mathrm{a}$ & $22 \mathrm{a}$ & $16.8 \mathrm{a}$ & $13.9 \mathrm{a}$ & $34.7 \mathrm{a}$ & $60.8 \mathrm{a}$ & $35.8 \mathrm{a}$ & $70.2 \mathrm{a}$ \\
\hline Control & $0.015 \mathrm{~d}$ & $12 \mathrm{~d}$ & $0.05 \mathrm{e}$ & $0.1 \mathrm{~d}$ & $11 \mathrm{~d}$ & $7.5 \mathrm{~d}$ & $9 \mathrm{~d}$ & $14 \mathrm{~d}$ & $25.4 \mathrm{f}$ & $15.5 \mathrm{c}$ & $27.4 \mathrm{e}$ \\
\hline
\end{tabular}

Table 4c. Means of Characters measured in calendula plants that are affected by micronutrients (400 ppm) concentration and control plants $\left(1^{\text {st }}\right.$ year $)$.

\begin{tabular}{cccccccccc}
\hline & $\begin{array}{c}\text { Shoot } \\
\text { Treatments }\end{array}$ & $\begin{array}{c}\text { Shoot } \\
\text { fresh } \\
\text { matter }\end{array}$ & $\begin{array}{c}\text { Root dry } \\
\text { matter }\end{array}$ & $\begin{array}{c}\text { Root } \\
\text { fresh } \\
\text { matter }\end{array}$ & $\begin{array}{c}\text { Number } \\
\text { of } \\
\text { flower }\end{array}$ & $\begin{array}{c}\text { Weight } \\
\text { of } \\
\text { flower }\end{array}$ & $\begin{array}{c}\text { Essence } \\
\text { percentage }\end{array}$ & phenols & Flavonoid \\
\hline Copper $(\mathrm{Cu})$ & $6.6 \mathrm{c}$ & $12.2 \mathrm{~d}$ & $7.1 \mathrm{~cd}$ & $14.1 \mathrm{de}$ & $21.1 \mathrm{de}$ & $7 \mathrm{~cd}$ & $0.24 \mathrm{~d}$ & $28.4 \mathrm{~d}$ & $14.1 \mathrm{c}$ \\
Manganese $(\mathrm{Mn})$ & $5.6 \mathrm{c}$ & $12.5 \mathrm{~cd}$ & $6.8 \mathrm{~cd}$ & $13.1 \mathrm{de}$ & $22.1 \mathrm{~d}$ & $7.5 \mathrm{~cd}$ & $0.22 \mathrm{~d}$ & $28.2 \mathrm{~d}$ & $14.2 \mathrm{c}$ \\
Iron $(\mathrm{Fe})$ & $7.3 \mathrm{bc}$ & $12.7 \mathrm{~cd}$ & $6.6 \mathrm{~cd}$ & $12.1 \mathrm{de}$ & $22.2 \mathrm{~d}$ & $8 \mathrm{c}$ & $0.25 \mathrm{~d}$ & $32.9 \mathrm{~d}$ & $14.4 \mathrm{c}$ \\
Boron $(\mathrm{B})$ & $6.1 \mathrm{c}$ & $12.2 \mathrm{~d}$ & $6.6 \mathrm{~cd}$ & $11.9 \mathrm{e}$ & $20.1 \mathrm{de}$ & $6.6 \mathrm{~d}$ & $0.24 \mathrm{~d}$ & $30.1 \mathrm{~d}$ & $15.1 \mathrm{c}$ \\
$\mathrm{Cu} \times \mathrm{Mn}$ & $7.2 \mathrm{bc}$ & $13.2 \mathrm{~cd}$ & $7.7 \mathrm{~cd}$ & $14.6 \mathrm{~d}$ & $22.4 \mathrm{~cd}$ & $9.1 \mathrm{~b}$ & $0.56 \mathrm{c}$ & $39.1 \mathrm{bc}$ & $15.5 \mathrm{c}$ \\
$\mathrm{Cu} \times \mathrm{Fe}$ & $7.4 \mathrm{bc}$ & $13.7 \mathrm{~cd}$ & $8.1 \mathrm{~cd}$ & $16.1 \mathrm{~cd}$ & $23.1 \mathrm{~cd}$ & $9.3 \mathrm{~b}$ & $0.52 \mathrm{c}$ & $38.4 \mathrm{bc}$ & $16.2 \mathrm{c}$ \\
$\mathrm{Cu} \times \mathrm{B}$ & $7.8 \mathrm{bc}$ & $14.1 \mathrm{~cd}$ & $9.1 \mathrm{c}$ & $17.9 \mathrm{c}$ & $24.4 \mathrm{~cd}$ & $9.4 \mathrm{~b}$ & $0.55 \mathrm{c}$ & $37 \mathrm{c}$ & $16.7 \mathrm{bc}$ \\
$\mathrm{Mn} \times \mathrm{Fe}$ & $8.1 \mathrm{bc}$ & $15.1 \mathrm{c}$ & $8.8 \mathrm{~cd}$ & $17.1 \mathrm{~cd}$ & $25.7 \mathrm{c}$ & $9.5 \mathrm{~b}$ & $0.59 \mathrm{bc}$ & $38.8 \mathrm{bc}$ & $19.5 \mathrm{~b}$ \\
$\mathrm{Mn} \times \mathrm{B}$ & $6.5 \mathrm{c}$ & $14.1 \mathrm{~cd}$ & $8.5 \mathrm{~cd}$ & $18.1 \mathrm{c}$ & $23.1 \mathrm{~cd}$ & $9.5 \mathrm{~b}$ & $0.54 \mathrm{c}$ & $38.1 \mathrm{c}$ & $18.5 \mathrm{~b}$ \\
$\mathrm{Fe} \times \mathrm{B}$ & $7.3 \mathrm{bc}$ & $16.6 \mathrm{bc}$ & $8.3 \mathrm{~cd}$ & $17.2 \mathrm{~cd}$ & $25.5 \mathrm{c}$ & $9.6 \mathrm{ab}$ & $0.62 \mathrm{bc}$ & $40.2 \mathrm{bc}$ & $18 \mathrm{bc}$ \\
$\mathrm{Cu} \times \mathrm{Mn} \times \mathrm{Fe}$ & $8.9 \mathrm{ab}$ & $16.7 \mathrm{bc}$ & $10.1 \mathrm{bc}$ & $21.1 \mathrm{bc}$ & $27.2 \mathrm{~b}$ & $10.2 \mathrm{a}$ & $0.71 \mathrm{~b}$ & $44.1 \mathrm{~b}$ & $20.1 \mathrm{~b}$ \\
$\mathrm{Cu} \times \mathrm{Mn} \times \mathrm{B}$ & $9.2 \mathrm{ab}$ & $17.1 \mathrm{bc}$ & $10.2 \mathrm{bc}$ & $21.3 \mathrm{bc}$ & $27.4 \mathrm{~b}$ & $10.4 \mathrm{a}$ & $0.7 \mathrm{~b}$ & $42.2 \mathrm{~b}$ & $22 \mathrm{~b}$ \\
$\mathrm{Cu} \times \mathrm{Fe} \times \mathrm{B}$ & $9.6 \mathrm{ab}$ & $17.4 \mathrm{bc}$ & $11.1 \mathrm{~b}$ & $23.1 \mathrm{~b}$ & $27.6 \mathrm{~b}$ & $10.4 \mathrm{a}$ & $0.8 \mathrm{ab}$ & $44 \mathrm{~b}$ & $24 \mathrm{ab}$ \\
$\mathrm{Fe} \times \mathrm{B} \times \mathrm{Mn}$ & $9.1 \mathrm{ab}$ & $18.1 \mathrm{~b}$ & $11.4 \mathrm{~b}$ & $23.7 \mathrm{~b}$ & $27.1 \mathrm{~b}$ & $10.1 \mathrm{a}$ & $0.8 \mathrm{ab}$ & $43 \mathrm{~b}$ & $23 \mathrm{~b}$ \\
$\mathrm{Cu} \times \mathrm{B} \times \mathrm{Mn} \times \mathrm{Fe}$ & $10.8 \mathrm{a}$ & $22.1 \mathrm{a}$ & $15.2 \mathrm{a}$ & $32.6 \mathrm{a}$ & $33.1 \mathrm{a}$ & $10.8 \mathrm{a}$ & $0.9 \mathrm{a}$ & $55.3 \mathrm{a}$ & $30 \mathrm{a}$ \\
$\mathrm{Control}$ & $5.1 \mathrm{c}$ & $10.3 \mathrm{e}$ & $6.1 \mathrm{~d}$ & $12.2 \mathrm{de}$ & $18.1 \mathrm{e}$ & $6.9 \mathrm{~d}$ & $0.21 \mathrm{~d}$ & $25.1 \mathrm{~d}$ & $12.5 \mathrm{c}$ \\
\hline
\end{tabular}


Table 4. continued Means of Characters measured in calendula plants that are affected by micronutrients (400 ppm) concentration and control plants ( $2^{\text {nd }}$ year $)$.

\begin{tabular}{|c|c|c|c|c|c|c|c|c|c|}
\hline Treatments & $\begin{array}{c}\text { Shoot dry } \\
\text { matter }\end{array}$ & $\begin{array}{l}\text { Shoot } \\
\text { fresh } \\
\text { matter }\end{array}$ & $\begin{array}{c}\text { Root dry } \\
\text { matter }\end{array}$ & $\begin{array}{l}\text { Root } \\
\text { fresh } \\
\text { matter }\end{array}$ & $\begin{array}{c}\text { Number } \\
\text { of } \\
\text { flower }\end{array}$ & $\begin{array}{c}\text { Weight } \\
\text { of } \\
\text { flower }\end{array}$ & $\begin{array}{c}\text { Essence } \\
\text { percentage }\end{array}$ & phenols & Flavonoid \\
\hline Copper $(\mathrm{Cu})$ & $6.8 \mathrm{c}$ & $12.4 \mathrm{~cd}$ & $7.3 \mathrm{~d}$ & $15.2 \mathrm{~cd}$ & $22.1 \mathrm{~cd}$ & $8 \mathrm{~d}$ & $0.3 \mathrm{de}$ & $28.6 \mathrm{~d}$ & $15.2 \mathrm{~d}$ \\
\hline Manganese(Mn) & $5.8 \mathrm{c}$ & $12.6 \mathrm{~cd}$ & $7 \mathrm{~d}$ & $14.2 \mathrm{~d}$ & $23.1 \mathrm{~cd}$ & $8.1 \mathrm{~d}$ & $0.31 \mathrm{de}$ & $28.4 \mathrm{~d}$ & $15.3 \mathrm{~d}$ \\
\hline Iron $(\mathrm{Fe})$ & $7.8 \mathrm{ab}$ & $12.8 \mathrm{~cd}$ & $6.8 \mathrm{~d}$ & $12.4 \mathrm{~d}$ & $23.2 \mathrm{~cd}$ & $9 \mathrm{c}$ & $0.34 \mathrm{de}$ & $33.1 \mathrm{~cd}$ & $14.7 \mathrm{~d}$ \\
\hline Boron (B) & $6.7 \mathrm{c}$ & $12.4 \mathrm{~cd}$ & $6.4 \mathrm{~d}$ & $12.5 \mathrm{~d}$ & $21.1 \mathrm{~d}$ & $7 \mathrm{~d}$ & $0.27 \mathrm{e}$ & $31.1 \mathrm{~d}$ & $15.5 \mathrm{~d}$ \\
\hline $\mathrm{Cu} \times \mathrm{Mn}$ & $7.7 \mathrm{ab}$ & $13.6 \mathrm{~cd}$ & $7.9 \mathrm{~cd}$ & $15.6 \mathrm{~cd}$ & $23.4 \mathrm{~cd}$ & $10.1 \mathrm{~b}$ & $0.57 \mathrm{c}$ & $39.5 \mathrm{bc}$ & $15.6 \mathrm{~d}$ \\
\hline $\mathrm{Cu} \times \mathrm{Fe}$ & $7.5 \mathrm{ab}$ & $13.9 \mathrm{~cd}$ & $8.6 \mathrm{~cd}$ & $16.5 \mathrm{~cd}$ & $24.1 \mathrm{c}$ & $10.2 \mathrm{~b}$ & $0.55 \mathrm{c}$ & $38.6 \mathrm{c}$ & $16.5 \mathrm{~d}$ \\
\hline $\mathrm{Cu} \times \mathrm{B}$ & $8.1 \mathrm{ab}$ & $12.1 \mathrm{~d}$ & $8.8 \mathrm{c}$ & $18.3 \mathrm{c}$ & $24.2 \mathrm{c}$ & $10 \mathrm{~b}$ & $0.56 \mathrm{c}$ & $37.5 \mathrm{c}$ & $16.9 \mathrm{~cd}$ \\
\hline $\mathrm{Mn} \times \mathrm{Fe}$ & $8.5 \mathrm{ab}$ & $16.1 \mathrm{c}$ & $9.1 \mathrm{c}$ & $18.4 \mathrm{c}$ & $26.4 b c$ & $10.2 \mathrm{~b}$ & $0.6 \mathrm{c}$ & $39.8 \mathrm{c}$ & $20.1 \mathrm{c}$ \\
\hline $\mathrm{Mn} \times \mathrm{B}$ & $6.7 \mathrm{c}$ & $14.2 \mathrm{c}$ & $8.7 \mathrm{~cd}$ & $18.7 \mathrm{c}$ & $25.1 \mathrm{bc}$ & $10.1 \mathrm{~b}$ & $0.57 \mathrm{c}$ & $38.5 \mathrm{c}$ & $19.9 \mathrm{c}$ \\
\hline $\mathrm{Fe} \times \mathrm{B}$ & $7.9 \mathrm{ab}$ & $16.7 \mathrm{bc}$ & $8.4 \mathrm{~cd}$ & $18.5 \mathrm{c}$ & $26.5 b c$ & $10.2 \mathrm{~b}$ & $0.64 \mathrm{c}$ & $41.2 \mathrm{bc}$ & $18.8 \mathrm{c}$ \\
\hline $\mathrm{Cu} \times \mathrm{Mn} \times \mathrm{Fe}$ & $9.3 \mathrm{~b}$ & $16.8 \mathrm{bc}$ & $11.2 \mathrm{~b}$ & $22.1 \mathrm{bc}$ & $28.2 \mathrm{~b}$ & $11.7 \mathrm{a}$ & $0.74 \mathrm{~b}$ & $45.1 \mathrm{~b}$ & $21.5 \mathrm{bc}$ \\
\hline $\mathrm{Cu} \times \mathrm{Mn} \times \mathrm{B}$ & $9.7 \mathrm{~b}$ & $17.3 \mathrm{bc}$ & $11.6 \mathrm{~b}$ & $22.4 \mathrm{bc}$ & $28.4 \mathrm{~b}$ & $11.6 \mathrm{a}$ & $0.73 \mathrm{~b}$ & $43.2 \mathrm{bc}$ & $22.3 \mathrm{bc}$ \\
\hline $\mathrm{Cu} \times \mathrm{Fe} \times \mathrm{B}$ & $10.1 \mathrm{ab}$ & $17.6 \mathrm{bc}$ & $11.4 \mathrm{~b}$ & $24.5 \mathrm{bc}$ & $28.6 \mathrm{~b}$ & $11.1 \mathrm{ab}$ & $0.82 \mathrm{~b}$ & $45.2 \mathrm{~b}$ & $24.5 \mathrm{~b}$ \\
\hline $\mathrm{Fe} \times \mathrm{B} \times \mathrm{Mn}$ & $10.1 \mathrm{ab}$ & $18.2 \mathrm{~b}$ & $11.8 \mathrm{~b}$ & $25.5 \mathrm{~b}$ & $28.1 \mathrm{~b}$ & $11.7 \mathrm{a}$ & $0.9 \mathrm{ab}$ & $45.1 \mathrm{~b}$ & $23.4 \mathrm{~b}$ \\
\hline $\mathrm{Cu} \times \mathrm{B} \times \mathrm{Mn} \times \mathrm{Fe}$ & $12.1 \mathrm{a}$ & $23.4 \mathrm{a}$ & $16.3 \mathrm{a}$ & $34.1 \mathrm{a}$ & $33.5 \mathrm{a}$ & $11.9 \mathrm{a}$ & $1 \mathrm{a}$ & $56.2 \mathrm{a}$ & $31.2 \mathrm{a}$ \\
\hline Control & $6.1 \mathrm{c}$ & $10.5 \mathrm{~d}$ & $6.7 \mathrm{~d}$ & $14 \mathrm{~d}$ & $18.3 \mathrm{~d}$ & $7.1 \mathrm{~d}$ & $0.22 \mathrm{e}$ & $26.1 \mathrm{~d}$ & $14.2 \mathrm{~d}$ \\
\hline
\end{tabular}

Table 4. continued Means of Characters measured in thyme plants that are affected by micronutrients (400 ppm) concentration and control plants ( $1^{\text {st }}$ year $)$.

\begin{tabular}{|c|c|c|c|c|c|c|c|c|c|}
\hline Treatments & $\begin{array}{c}\text { Shoot } \\
\text { dry } \\
\text { matter }\end{array}$ & $\begin{array}{l}\text { Shoot } \\
\text { fresh } \\
\text { matter }\end{array}$ & $\begin{array}{c}\text { Root dry } \\
\text { matter }\end{array}$ & $\begin{array}{l}\text { Root } \\
\text { fresh } \\
\text { matter }\end{array}$ & Height & $\begin{array}{l}\text { Number } \\
\text { of stems }\end{array}$ & $\begin{array}{c}\text { Essence } \\
\text { percentage }\end{array}$ & $\begin{array}{l}\text { Carvacrol } \\
\text { percentage }\end{array}$ & $\begin{array}{c}\text { Thymol } \\
\text { percentage }\end{array}$ \\
\hline Copper $(\mathrm{Cu})$ & $6.6 \mathrm{c}$ & $12.2 \mathrm{~d}$ & $7.1 \mathrm{~cd}$ & $14.1 \mathrm{de}$ & $21.1 \mathrm{de}$ & $7 \mathrm{~cd}$ & $0.24 \mathrm{~d}$ & $28.4 \mathrm{~d}$ & $14.1 \mathrm{c}$ \\
\hline Manganese(Mn) & $5.6 \mathrm{c}$ & $12.5 \mathrm{~cd}$ & $6.8 \mathrm{~cd}$ & $13.1 \mathrm{de}$ & $22.1 \mathrm{~d}$ & $7.5 \mathrm{~cd}$ & $0.22 \mathrm{~d}$ & $28.2 \mathrm{~d}$ & $14.2 \mathrm{c}$ \\
\hline Iron $(\mathrm{Fe})$ & $7.3 \mathrm{bc}$ & $12.7 \mathrm{~cd}$ & $6.6 \mathrm{~cd}$ & $12.1 \mathrm{de}$ & $22.2 \mathrm{~d}$ & $8 \mathrm{c}$ & $0.25 \mathrm{~d}$ & $32.9 \mathrm{~d}$ & $14.4 \mathrm{c}$ \\
\hline Zinc $(\mathrm{Zn})$ & $6.1 \mathrm{c}$ & $12.2 \mathrm{~d}$ & $6.6 \mathrm{~cd}$ & $11.9 \mathrm{e}$ & $20.1 \mathrm{de}$ & $6.6 \mathrm{~d}$ & $0.24 \mathrm{~d}$ & $30.1 \mathrm{~d}$ & $15.1 \mathrm{c}$ \\
\hline $\mathrm{Cu} \times \mathrm{Mn}$ & $7.2 \mathrm{bc}$ & $13.2 \mathrm{~cd}$ & $7.7 \mathrm{~cd}$ & $14.6 \mathrm{~d}$ & $22.4 \mathrm{~cd}$ & $9.1 \mathrm{~b}$ & $0.56 \mathrm{c}$ & $39.1 \mathrm{bc}$ & $15.5 \mathrm{c}$ \\
\hline $\mathrm{Cu} \times \mathrm{Fe}$ & $7.4 \mathrm{bc}$ & $13.7 \mathrm{~cd}$ & $8.1 \mathrm{~cd}$ & $16.1 \mathrm{~cd}$ & $23.1 \mathrm{~cd}$ & $9.3 \mathrm{~b}$ & $0.52 \mathrm{c}$ & $38.4 \mathrm{bc}$ & $16.2 \mathrm{c}$ \\
\hline $\mathrm{Cu} \times \mathrm{Zn}$ & $7.8 \mathrm{bc}$ & $14.1 \mathrm{~cd}$ & $9.1 \mathrm{c}$ & $17.9 \mathrm{c}$ & $24.4 \mathrm{~cd}$ & $9.4 \mathrm{~b}$ & $0.55 \mathrm{c}$ & $37 \mathrm{c}$ & $16.7 \mathrm{bc}$ \\
\hline $\mathrm{Mn} \times \mathrm{Fe}$ & $8.1 \mathrm{bc}$ & $15.1 \mathrm{c}$ & $8.8 \mathrm{~cd}$ & $17.1 \mathrm{~cd}$ & $25.7 \mathrm{c}$ & $9.5 \mathrm{~b}$ & $0.59 \mathrm{bc}$ & $38.8 \mathrm{bc}$ & $19.5 \mathrm{~b}$ \\
\hline $\mathrm{Mn} \times \mathrm{Zn}$ & $6.5 \mathrm{c}$ & $14.1 \mathrm{~cd}$ & $8.5 \mathrm{~cd}$ & $18.1 \mathrm{c}$ & $23.1 \mathrm{~cd}$ & $9.5 \mathrm{~b}$ & $0.54 \mathrm{c}$ & $38.1 \mathrm{c}$ & $18.5 \mathrm{~b}$ \\
\hline $\mathrm{Fe} \times \mathrm{Zn}$ & $7.3 \mathrm{bc}$ & $16.6 \mathrm{bc}$ & $8.3 \mathrm{~cd}$ & $17.2 \mathrm{~cd}$ & $25.5 \mathrm{c}$ & $9.6 \mathrm{ab}$ & $0.62 \mathrm{bc}$ & $40.2 \mathrm{bc}$ & $18 \mathrm{bc}$ \\
\hline $\mathrm{Cu} \times \mathrm{Mn} \times \mathrm{Fe}$ & $8.9 \mathrm{ab}$ & $16.7 \mathrm{bc}$ & $10.1 \mathrm{bc}$ & $21.1 \mathrm{bc}$ & $27.2 \mathrm{~b}$ & $10.2 \mathrm{a}$ & $0.71 \mathrm{~b}$ & $44.1 \mathrm{~b}$ & $20.1 \mathrm{~b}$ \\
\hline $\mathrm{Cu} \times \mathrm{Mn} \times \mathrm{Zn}$ & $9.2 \mathrm{ab}$ & $17.1 \mathrm{bc}$ & $10.2 \mathrm{bc}$ & $21.3 \mathrm{bc}$ & $27.4 \mathrm{~b}$ & $10.4 \mathrm{a}$ & $0.7 \mathrm{~b}$ & $42.2 \mathrm{~b}$ & $22 \mathrm{~b}$ \\
\hline $\mathrm{Cu} \times \mathrm{Fe} \times \mathrm{Zn}$ & $9.6 \mathrm{ab}$ & $17.4 \mathrm{bc}$ & $11.1 \mathrm{~b}$ & $23.1 \mathrm{~b}$ & $27.6 \mathrm{~b}$ & $10.4 \mathrm{a}$ & $0.8 \mathrm{ab}$ & $44 \mathrm{~b}$ & $24 \mathrm{ab}$ \\
\hline $\mathrm{Fe} \times \mathrm{Zn} \times \mathrm{Mn}$ & $9.1 \mathrm{ab}$ & $18.1 \mathrm{~b}$ & $11.4 \mathrm{~b}$ & $23.7 \mathrm{~b}$ & $27.1 \mathrm{~b}$ & $10.1 \mathrm{a}$ & $0.8 \mathrm{ab}$ & $43 \mathrm{~b}$ & $23 \mathrm{~b}$ \\
\hline $\mathrm{Cu} \times \mathrm{Zn} \times \mathrm{Mn} \times \mathrm{Fe}$ & $10.8 \mathrm{a}$ & $22.1 \mathrm{a}$ & $15.2 \mathrm{a}$ & $32.6 \mathrm{a}$ & $33.1 \mathrm{a}$ & $10.8 \mathrm{a}$ & $0.9 \mathrm{a}$ & $55.3 \mathrm{a}$ & $30 \mathrm{a}$ \\
\hline Control & $5.1 \mathrm{c}$ & $10.3 \mathrm{e}$ & $6.1 \mathrm{~d}$ & $12.2 \mathrm{de}$ & $18.1 \mathrm{e}$ & $6.9 \mathrm{~d}$ & $0.21 \mathrm{~d}$ & $25.1 \mathrm{~d}$ & $12.5 \mathrm{c}$ \\
\hline
\end{tabular}


Table 4. continued Means of Characters measured in thyme plants that are affected by micronutrients (400 ppm) concentration and control plants $\left(2^{\text {nd }}\right.$ year $)$.

\begin{tabular}{|c|c|c|c|c|c|c|c|c|c|}
\hline Treatments & $\begin{array}{l}\text { Shoot dry } \\
\text { matter }\end{array}$ & $\begin{array}{l}\text { Shoot } \\
\text { fresh } \\
\text { matter }\end{array}$ & $\begin{array}{c}\text { Root dry } \\
\text { matter }\end{array}$ & $\begin{array}{l}\text { Root } \\
\text { fresh } \\
\text { matter }\end{array}$ & Height & $\begin{array}{l}\text { Number } \\
\text { of stems }\end{array}$ & $\begin{array}{c}\text { Essence } \\
\text { percentage }\end{array}$ & $\begin{array}{l}\text { Carvacrol } \\
\text { percentage }\end{array}$ & $\begin{array}{c}\text { Thymol } \\
\text { percentage }\end{array}$ \\
\hline Copper $(\mathrm{Cu})$ & $6.8 \mathrm{c}$ & $12.4 \mathrm{~cd}$ & $7.3 \mathrm{~d}$ & $15.2 \mathrm{~cd}$ & $22.1 \mathrm{~cd}$ & $8 \mathrm{~d}$ & $0.3 \mathrm{de}$ & $28.6 \mathrm{~d}$ & $15.2 \mathrm{~d}$ \\
\hline Manganese(Mn) & $5.8 \mathrm{c}$ & $12.6 \mathrm{~cd}$ & $7 \mathrm{~d}$ & $14.2 \mathrm{~d}$ & $23.1 \mathrm{~cd}$ & $8.1 \mathrm{~d}$ & $0.31 \mathrm{de}$ & $28.4 \mathrm{~d}$ & $15.3 \mathrm{~d}$ \\
\hline Iron $(\mathrm{Fe})$ & $7.8 \mathrm{ab}$ & $12.8 \mathrm{~cd}$ & $6.8 \mathrm{~d}$ & $12.4 \mathrm{~d}$ & $23.2 \mathrm{~cd}$ & $9 \mathrm{c}$ & $0.34 \mathrm{de}$ & $33.1 \mathrm{~cd}$ & $14.7 \mathrm{~d}$ \\
\hline $\operatorname{Zinc}(\mathrm{Zn})$ & $6.7 \mathrm{c}$ & $12.4 \mathrm{~cd}$ & $6.4 \mathrm{~d}$ & $12.5 \mathrm{~d}$ & $21.1 \mathrm{~d}$ & $7 \mathrm{~d}$ & $0.27 \mathrm{e}$ & $31.1 \mathrm{~d}$ & $15.5 \mathrm{~d}$ \\
\hline $\mathrm{Cu} \times \mathrm{Mn}$ & $7.7 \mathrm{ab}$ & $13.6 \mathrm{~cd}$ & $7.9 \mathrm{~cd}$ & $15.6 \mathrm{~cd}$ & $23.4 \mathrm{~cd}$ & $10.1 \mathrm{~b}$ & $0.57 \mathrm{c}$ & $39.5 \mathrm{bc}$ & $15.6 \mathrm{~d}$ \\
\hline $\mathrm{Cu} \times \mathrm{Fe}$ & $7.5 \mathrm{ab}$ & $13.9 \mathrm{~cd}$ & $8.6 \mathrm{~cd}$ & $16.5 \mathrm{~cd}$ & $24.1 \mathrm{c}$ & $10.2 \mathrm{~b}$ & $0.55 \mathrm{c}$ & $38.6 \mathrm{c}$ & $16.5 \mathrm{~d}$ \\
\hline $\mathrm{Cu} \times \mathrm{Zn}$ & $8.1 \mathrm{ab}$ & $12.1 \mathrm{~d}$ & $8.8 \mathrm{c}$ & $18.3 \mathrm{c}$ & $24.2 \mathrm{c}$ & $10 \mathrm{~b}$ & $0.56 \mathrm{c}$ & $37.5 \mathrm{c}$ & $16.9 \mathrm{~cd}$ \\
\hline $\mathrm{Mn} \times \mathrm{Fe}$ & $8.5 \mathrm{ab}$ & $16.1 \mathrm{c}$ & $9.1 \mathrm{c}$ & $18.4 \mathrm{c}$ & $26.4 \mathrm{bc}$ & $10.2 \mathrm{~b}$ & $0.6 \mathrm{c}$ & $39.8 \mathrm{c}$ & $20.1 \mathrm{c}$ \\
\hline $\mathrm{Mn} \times \mathrm{Zn}$ & $6.7 \mathrm{c}$ & $14.2 \mathrm{c}$ & $8.7 \mathrm{~cd}$ & $18.7 \mathrm{c}$ & $25.1 \mathrm{bc}$ & $10.1 \mathrm{~b}$ & $0.57 \mathrm{c}$ & $38.5 \mathrm{c}$ & $19.9 \mathrm{c}$ \\
\hline $\mathrm{Fe} \times \mathrm{Zn}$ & $7.9 \mathrm{ab}$ & $16.7 \mathrm{bc}$ & $8.4 \mathrm{~cd}$ & $18.5 \mathrm{c}$ & $26.5 \mathrm{bc}$ & $10.2 \mathrm{~b}$ & $0.64 \mathrm{c}$ & $41.2 \mathrm{bc}$ & $18.8 \mathrm{c}$ \\
\hline $\mathrm{Cu} \times \mathrm{Mn} \times \mathrm{Fe}$ & $9.3 \mathrm{~b}$ & $16.8 \mathrm{bc}$ & $11.2 \mathrm{~b}$ & $22.1 \mathrm{bc}$ & $28.2 \mathrm{~b}$ & $11.7 \mathrm{a}$ & $0.74 \mathrm{~b}$ & $45.1 \mathrm{~b}$ & $21.5 \mathrm{bc}$ \\
\hline $\mathrm{Cu} \times \mathrm{Mn} \times \mathrm{Zn}$ & $9.7 \mathrm{~b}$ & $17.3 \mathrm{bc}$ & $11.6 \mathrm{~b}$ & $22.4 \mathrm{bc}$ & $28.4 \mathrm{~b}$ & $11.6 \mathrm{a}$ & $0.73 \mathrm{~b}$ & $43.2 \mathrm{bc}$ & $22.3 \mathrm{bc}$ \\
\hline $\mathrm{Cu} \times \mathrm{Fe} \times \mathrm{Zn}$ & $10.1 \mathrm{ab}$ & $17.6 \mathrm{bc}$ & $11.4 \mathrm{~b}$ & $24.5 \mathrm{bc}$ & $28.6 \mathrm{~b}$ & $11.1 \mathrm{ab}$ & $0.82 \mathrm{~b}$ & $45.2 \mathrm{~b}$ & $24.5 \mathrm{~b}$ \\
\hline $\mathrm{Fe} \times \mathrm{Zn} \times \mathrm{Mn}$ & $10.1 \mathrm{ab}$ & $18.2 \mathrm{~b}$ & $11.8 \mathrm{~b}$ & $25.5 \mathrm{~b}$ & $28.1 \mathrm{~b}$ & $11.7 \mathrm{a}$ & $0.9 \mathrm{ab}$ & $45.1 \mathrm{~b}$ & $23.4 \mathrm{~b}$ \\
\hline $\mathrm{Cu} \times \mathrm{Zn} \times \mathrm{Mn} \times \mathrm{Fe}$ & $12.1 \mathrm{a}$ & $23.4 \mathrm{a}$ & $16.3 \mathrm{a}$ & $34.1 \mathrm{a}$ & $33.5 \mathrm{a}$ & $11.9 \mathrm{a}$ & $1 \mathrm{a}$ & $56.2 \mathrm{a}$ & $31.2 \mathrm{a}$ \\
\hline Control & $6.1 \mathrm{c}$ & $10.5 \mathrm{~d}$ & $6.7 \mathrm{~d}$ & $14 \mathrm{~d}$ & $18.3 \mathrm{~d}$ & $7.1 \mathrm{~d}$ & $0.22 \mathrm{e}$ & $26.1 \mathrm{~d}$ & $14.2 \mathrm{~d}$ \\
\hline
\end{tabular}

\section{Discussion}

Results showed beneficial micronutrient application on medicinal plants (Table 2). Increasing of micronutrients can obtain more yield, essence and essential oils from calendula (Naguib et al. 2005). The beneficial application of micronutrients was reported by other researchers (Yadegari et al. 2012; Galavi et al. 2012). Results of this research showed that foliar application of micronutrient made the more essential oil in flower of medicinal plants (Tables 2, 4). A similar effect of micronutrient supply on this parameter was also reported on M.chamomilla (Nasiri et al. 2010), Coriandrum sativum (Said-Al Ahland Omer, 2009), Menthasp (Rawia et al. 2010) and Ocimum basilicum (Said-Al Ahland Mahmoud, 2010). In the present study, the effect of micronutrients applications was determined on the growth and yield of medicinal plants in two consecutive years.
Dry matter flower increased in both years with micronutrients applications. The essential oil yield increased with $\mathrm{Fe}^{2+}, \mathrm{Cu}^{2+}, \mathrm{Zn}^{2+}$ and $\mathrm{Mn}^{2+}$ applications because there was a significant increase in dry matter and number of flower. This is the first report in which the effects of $\mathrm{Fe}^{2+}, \mathrm{Cu}^{2+}, \mathrm{Zn}^{2+}$ and $\mathrm{Mn}^{2+}$ applications on certain physiological characteristics were determined for medicinal plants and also their relationship with yield and essential oil content. It is obvious that weight and number of flowers can be used as a tool for selecting new cultivars with high yield. It seems the weight of shoot dry matter was the best character that correlated by most of characters a finding with which others agree (Mhamdi et al. 2009; Naghdi Badi and Sorooshzadeh, 2011; Babaeian et al. 2012; Samia and Mohmoud, 2009; Jamali et al. 2014). From the present data, Its concluded that applications of $\mathrm{Fe}^{2+}$, $\mathrm{Cu}^{2+}, \mathrm{Zn}^{2+}$ and $\mathrm{Mn}^{2+}$ are beneficial for medicinal plants with concentrations of 400ppm or lower, and 
can result in an increase in dry matter yield of up to $40 \% . \mathrm{Fe}^{2+}, \mathrm{Cu}^{2+}, \mathrm{Zn}^{2+}$ and $\mathrm{Mn}^{2+}$ have direct functions on growth and the development of plants. There are still many unanswered questions about how $\mathrm{Fe}^{2+}$, $\mathrm{Cu}^{2+}, \mathrm{Zn}^{2+}$ and $\mathrm{Mn}^{2+}$ act in increasing yield and its components (number of flower plant) for medicinal plants. One possibility is that the foliarly applied $\mathrm{Fe}^{2+}$, $\mathrm{Cu}^{2+}, \mathrm{Zn}^{2+}$ and $\mathrm{Mn}^{2+}$ can affect dry matter accumulation and increase dry matter flower. Micronutrients can increase the weight of shoot dry matter and weight of root dry matter (Younis et al. 2013; Yarnia et al. 2012). It seem, control plants that no have foliar application, were better than plants that have upper concentration of micronutrients. Combinations of micronutrients upper concentration than $400 \mathrm{ppm}$ had more reducer effect than single of them. These results reflect the role of applying the four foliar fertilizers in improving the total essential oils in medicinal plants. It seems that $\mathrm{Fe}, \mathrm{Cu}, \mathrm{Mn}$ and $\mathrm{Zn}$ by the effect on absorption and transition of essential nutrients made the change of metabolism and growth and development and then increased upper phytochemicals. Generally, the obtained results revealed that under Iranian conditions applying foliar fertilizer as $\mathrm{Fe}, \mathrm{Cu}, \mathrm{Mn}$ and $\mathrm{Zn}$ at $400 \mathrm{ppm}$ resulted in highest improvement of growth character, yield and chemical constituents. Decreasing effect of $\mathrm{Fe}_{4} \mathrm{Cu}_{4} \mathrm{Mn}_{4} \mathrm{Zn}_{4}$ made the equivalent amount of control plants.

\section{Conclusion}

Plants treated with $400 \mathrm{ppm}$ of Iron, Zinc, Manganese and Copper, had the more fresh and dry weight of flowers and essence percentage. It could be concluded from the results that Iron, Zinc, Manganese and Copper fertilization had significant effects on measured characters as well as the chemical component of the essence of medicinal plants. The most suitable Fe$\mathrm{Zn}-\mathrm{Mn}-\mathrm{Cu}$ supply for production of medicinal plants to obtain the highest number and weight of flowers in Shahrekord, Iran is 400ppm foliar application of micronutrients. Effects of combined application of micronutrients fertilizers is suggested in compare with the separately use of them.

Results obtained from this study showed that the essential oils composition of lemon balm was significantly affected by foliar application of micronutrients. The highest amount of flavonoids, phenols, carotenoids in Borago officinalis and Calendula officinalis L.; carvacrol and thymol percentage, in Thymus vulgaris L., obtained from $400 \mathrm{ppm}$ of micronutrients foliar application in two cultivation seasons. This study provides some useful information about the efficacy of foliar application of micronutrients in soils with undesirable characteristics, and chemical properties in particular. Since in some cases, these methods are relatively new and need further refinement with regard to rates, timing, and technique.

\section{References}

Adams, R.P. 2001. Identification of Essential Oil Components by Gas Chromatography/ Mass Spectroscopy. Allured publishing Corp. Carol Stream. USA.

Babaeian, M., Esmaeilian, Y., Tavassoli, A.,Asgharzade, A. 2012. Efficacy of different iron, zinc and magnesium fertilizers on yield and yield components of barley. Afr. J. Microbiol. Res. 6, 5754-56.

Babaeian, M., Tavassoli, A., Ghanbari, A., Esmaeilian, Y., Fahimifard, M. 2012. Effects of foliar micronutrient application on osmotic adjustments, grain yield and yield components in sunflower under water stress at three stages. Afr. J. Agric. Res. 6, 1204-08. 
Borghei, M., Arjmandi, R., Moogouei, R. 2011. Potential of Calendula alata for phytoremediation of stable cesium and lead from solutions. Environmental Monito Assessment. 181, 63-68.

Bunghez, I.R., Ion, R.M. 2011. Complex spectral characterization of active principles from marigold (Calendula officinalis). Journal of Science and Arts. 1, 59-64.

Datta, J.K., Kundu, A., Dilwar, S., Mondal, N.K. 2011. Studies on the impact of micronutrient on germination, seedling growth and physiology of Bengal Gram under laboratory condition. Asian Journal of Crop Science. 3(2): 55-67.

Deletre, E., Chandre, F., Williams, L., Duménil1, C., Menut, C., Martin, T. 2015. Electrophysiological and behavioral characterization of bioactive compounds of the Thymus vulgaris, Cymbopogon winterianus, Cuminum cyminum and Cinnamomum zeylanicum essential oils against Anopheles gambiae and prospects for their use as bed net treatments. 8:316. DOI 10.1186/s13071015-0934-y.

Eberle, C.A., Frank, Forcella., Russ. Gesch., Dean, Peterson., James, Eklund. 2014. Seed germination of calendula in response to temperature. Industrial Crops and Products. 52, 199-204.

FriekeKuper, C., Marcel, V.W. Wijnands. 2014. Thymus. Encyclopedia of Immunotoxicology. DOI 10.1007/978-3-642-27786-3-1472-2.

Galavi, M., Ramroudi, M.,Tavassoli, A. 2012. Effect of micronutrients foliar application on yield and seed oil content of safflower (Carthamus tinctorius). Afr. J. Agric. Res. 7, 482-86.

Gilbertson, P.K., Berti, M.T., Johnson, B.L. 2014 Borage cardinal germination temperatures and seed development. Industrial Crops and Products. 59, 202-209.

Habib, M. 2012. Effect of supplementary nutrition with $\mathrm{Fe}, \mathrm{Zn}$ chelates and urea on wheat quality and quantity. African Journal Biotechnology. 11, 2661-65.

Heidari, M., Galavi, M., Hassani, M. 2011. Effect of sulfur and iron fertilizers on yield, yield components and nutrient uptake in sesame (Sesamum indicum L.) under water stress. African Journal Biotechnology. 10, 8816-22.

Heidarian, A.R., Kord, H., Mostafavi, Kh., Amini Mashhadi, F. 2011. Investigating $\mathrm{Fe}$ and $\mathrm{Zn}$ foliar application on yield and its components of soybean [Glycine $\max (\mathrm{L}$.) Merr.] at different growth stages. Journal Agriculture Biotechnology and Sustainable Development. 3, 189-97.

Jamali, C.A., Kasrati, A., Bekkouche, Kh., Hassani, L.,Wohlmuth, H., Leach, D., Abbad, A. 2014. Cultivation and the application of inorganic fertilizer modifies essential oil composition in two Moroccan species of Thymus. Industrial Crops and Products. 62, 113-118.

Khan, I., Zaman, M., Khan, M.J., Iqbal, M., Babar, M.N. 2014. How to improve yield and quality of potatoes: effects of two rates of urea $\mathrm{N}$, urease inhibitor and Cytozyme nutritional program. Journal of Soil Science and Plant Nutrition. 14 (2): 268-276. http://dx.doi.org/10.4067/S071895162014005000022 .

Lim, T.K. 2014. Calendula officinalis: Edible Medicinal And Non-Medicinal Plants. Volume 7, Flowers. DOI 10.1007/978-94-007-7359-0-15

Lin, L., Liu, L., Chiang, L., Lin, C. 2002. In-vitro antihepatoma activity of fifteen natural medicines from Canada. Phytotherapy Research. 16, 440-444.

Mello Prado, R., Ferreira Frade Junior, E., Rinaldi Mouta, E.,Cássia Gomes São João, A., Savério Souza Costa, R. 2007. Initial growth and nutritional condition of the wheat seedlings after application of zinc in the seeds. R.C. Suelo Nutr. Veg.7(2):22-31. ISSN 0718-2791. http://dx.doi. org/10.4067/S0718-27912007000200003. 
Mendes, M.D., Figueiredo, A.C., Oliveira, M.M., Trindade, H . 2014. Influence of culture media and fungal extracts on essential oils composition and on terpene synthase gene expression in Thymus caespititius. Plant Cell, Tissue and Organ Culture. 118, 457-469.

Mhamdi, B., Aidi Wannes, W., Sriti, J., Jellali, I., Ksouri, R., Marzouk, B. 2009. Effect of harvesting time on phenolic compounds and antiradical scavenging activity of Borago officinalis seed extracts. Industrial Crops and Products. 31, e1-e4.

Naghdi Badi, H., Sorooshzadeh, A. 2011. Evaluating potential of borage (Borago officinalis L.) in bioremediation of saline soil. African Journal of Biotechnology. 10 (2): 146-153.

Naguib, N.Y., Khalil, M.Y., El Sherbeny, S.E. 2005. A Comparative Study on the Productivity and Chemical Constituents of Various Sources and Species of Calendula Plants as Affected by Two Foliar Fertilizers. Journal of Applied Sciences Research. 1(2): 176-189.

Nasiri, Y., Zehtab-Salmasi, S.,Nasrullahzadeh, S., Najafi, N. 2010. Effects of foliar application of micronutrients ( $\mathrm{Fe}$ and $\mathrm{B}$ ) on flower yield and essential oil of chamomile (Matricaria chamomilla L.). Journal of Medicinal Plants Research. 4(17): 1733-1737.

Orioli Junior, V., Mello Prado, R., Luarte Leonel, C., Amélio Cazetta, D., Martoreli da Silveira, C., Josemara Barbosa Queiroz, R., Cristina Húngaro, J. 2008. Methods of zinc application in the nutrition and production of wheat plant dry matter. R.C. Suelo Nutr. Veg. 8(1): 28-36. http:// dx.doi.org/10.4067/S0718-27912008000100003.

Quezada, C., Vidal, I., Lemus, L., Sanchez, H. 2007. Effect of nitrogen fertilization on yield and fruit quality of raspberries (Rubusidaeus L.) under two fertigation programs. R.C. Suelo Nutr. Veg. 7(3): 1-15. http://dx.doi.org/10.4067/S071827912007000300001.
RatzLyko, A., Herman, A., Arct, J., Katarzyna, P. 2014. Evaluation of antioxidant and antimicrobial activities of Oenothera biennis, Borago officinalis, and Nigella sativa seedcake extracts. Food Sci. Biotechnol. 23(4):1029-1036.

Raza, M.A.S., Saleem, M.F., Shah, G.M., Khan, I.H., Raza, A. 2014. Exogenous application of glycinebetaine and potassium for improving water relations and grain yield of wheat under drought. Journal of Soil Science and Plant Nutrition. 14(2), 348-364. http://dx.doi.org/10.4067/S071895162014005000028 .

Rawia, A., Eid, R.K.M., Shaaban, S.H.A. 2010. Effect of foliar application of zinc and benzyladenine on growth, yield and chemical constituents of tuberose plants. Res. J. Agric. and Biol. Sci. 6, 732-43.

Said-Al Ahl, H.A.H.,Omer, E.A. 2009. Effect of spraying with Boron and / or iron on growth and chemical composition of coriander (Coriandrum sativum L.) harvested at three stages of development. Journal Medicine Food Plants. 1(2): 30-46.

Said-Al Ahl, H.A.H., Mahmoud, A. 2010. Effect of Boron and / or iron foliar application on growth and essential oil of sweet basil (Ocimum basilicum L.) under salt stress. Ozean Journal Applied Science. 3, 97-111.

Samia, M.Z., Mohmoud, A.M.A. 2009. Effects of corms storage, zinc application and their interaction on vegetative growth, flowering, corms productivity and chemical constituents of Tritonia crocata Ker Gawl Plant. J. Agric. Res. Kafr El-Sheikh Univ. 35, 230-55.

Sandana, P., Pinochet, D. 2014. Grain yield and phosphorus use efficiency of wheat and pea in a high yielding environment. J. Soil Sci. Plant Nutr.14 (4): 973-986. ISSN 0718-9516. http:// dx.doi.org/10.4067/S0718-95162014005000076. 
Schönherr, J., Fernandez, V., Schreiber, L. 2005. Rates of cuticular penetration of chelated FeIII: role of humidity, concentration, adjuvants, temperature, and type of chelate. Journal Agriculture Food Chemistry. 53, 4484-4492.

Sedghi, M., Pirzad, A., Amanpour, B. 2011. Light Absorption and Carotenoids Synthesis of Pot Marigold (Calendula officinalis L.) in Response to Phosphorous and Potassium Varying Levels. Academic pres. Not Sci Biol. 3(1):46-50.

Tyler, V.E. 1993. The Honest Herbal, third ed. Haworth Press, New York, pp: 51-53.

Wafa, A., Al-Khamees, A., Michael, D., Alrashdi, S., Adam, D., Algren, B., Morgan, W. 2011. Status Epilepticus Associated with Borage Oil Ingestion. J. Med. Toxicol. 7,154-157.

Wettasinghe, M., Shahidi, F., Amarowicz, R., Mamdouh, M. 2001. Phenolic acids in defatted seeds of borage (Borago officinalis L.). Food Chemistry. 75, 49-56.

Yadegari, M., Farahani, G.H.N., Mosadeghzad, Z. 2012. Biofertilizers effects on quantitative and qualitative yield of Thyme (Thymus vulgaris). African Journal of Agricultural Research. 7(34): 4716-4723.
Yañez-Mansilla, E., Cartes, P; Reyes-Díaz, M., Ribera-Fonseca, A., Rengel, Z., Alberdi, M. 2015. Leaf nitrogen thresholds ensuring high antioxidant features of Vaccinium corymbosum cultivars. J. Soil Sci. Plant Nutr. 15 (3):574-586.

Yarnia, M., Farzanian, M., Aliasgharzad, N. 2012. Effects of microelement fertilizers and phosphate biological fertilizer on some morphological traits of purple coneflower in water stress condition. African Journal of Microbiology Research. 6, 4825-32.

Younis, A., Riaz, A., Sajid, M., Mushtaq, N., Ahsan, M., Nadeem, M. 2013. Foliar application of macro- and micronutrients on the yield and quality of Rosa hybridacvs. Cardinal and Whisky Mac. African Journal of Biotechnology. 12(7): 702-708.

Wyllyam Do Vale, D., De Mello Prado, R., Natale, W., Ursulino Alves, A. 2008. Nutritional response of rootstock of lemon 'citrumelo' to nitrogen, phosphorus and potassium application. R.C.Suelo Nutr. Veg. 8, 40-48. 